\title{
Drosophila homolog of APP-BP1 (dAPP-BP1) interacts antagonistically with APPL during Drosophila development
}

\author{
H-J Kim ${ }^{1}$, S-H Kim ${ }^{1}$, S-O Shim ${ }^{1,3}$, E Park ${ }^{1}$, C Kim ${ }^{4}, K_{\text {Kim}}{ }^{1}$, MA Tanouye ${ }^{2,3}$ and J Yim ${ }^{\star, 1}$
}

$\beta$-Amyloid precursor protein binding protein 1 (APP-BP1) was previously identified based on its binding to the carboxyl terminal of $\beta$-amyloid precursor protein. In this report, we have discovered that a mutation of dAPP-BP1 (Drosophila ortholog of APP-BP1) hinders tissue development, causes apoptosis in imaginal disc cells, and blocks the NEDD8 conjugation pathway. We show that dAPP-BP1 specifically binds the intracellular domain of APP-like protein (APPL). The dAPP-BP1 mutation partially suppresses the abnormal macrochaete phenotype of $A p p I^{d}$, while overexpression of dAPP-BP1 causes abnormal macrochaetes. When APPL is overexpressed, the normal bristle pattern in the fly thorax is disturbed and apoptosis is induced in wing imaginal discs. APPL overexpression phenotypes are enhanced by reducing the level of AAPP-BP1. APPL overexpression is shown to inhibit the NEDD8 conjugation pathway. APPL-induced apoptosis is rescued by overexpression of dAPP-BP1. Our data suggest that APPL and dAPP-BP1 interact antagonistically during Drosophila development.

Cell Death and Differentiation (2007) 14, 103-115. doi:10.1038/sj.cdd.4401935; published online 21 April 2006

$\beta$-Amyloid precursor protein binding protein 1 (APP-BP1) was originally identified as a protein that interacts with the intracellular carboxyl terminal of APP ( $\beta$-amyloid precursor protein), thought to play a critical role in Alzheimer's disease (AD). ${ }^{1}$ APP-BP1 is homologous to the amino $(\mathrm{N})$ terminus of the ubiquitin-activating enzyme $E 1$, but lacks the conserved cysteine required for ubiquitin activation. APP-BP1 forms a heterodimer with hUba3. The hUba3 subunit is homologous to the carboxyl-terminus of E1 and apparently contributes the conserved cysteine to the dimer. The APP-BP1/hUba3 heterodimer provides E1-like functions in NEDD8 (ubiquitinlike protein) activation. Activated NEDD8 forms a thiol ester bond with the conjugating enzyme hUbc12, analogous to E2 in the ubiquitination pathway. Target proteins are covalently bound at a lysine residue. ${ }^{2}$ In mammalian cell lines, APP-BP1 drives the $\mathrm{S}$ - to $\mathrm{M}$-phase transition in dividing cells. When overexpressed, it can cause apoptosis in primary neurons. Apoptosis can be blocked by inhibition of the NEDD8 conjugation pathway. ${ }^{3}$ In AD patients, brain APP-BP1 protein levels in lipid rafts are increased. ${ }^{4}$

Well-characterized substrates described for the NEDD8 conjugation pathway (neddylation) mainly include only members of the cullin family of proteins. Every cullin family member is known to be modified by NEDD $8,{ }^{5}$ except for APC2. Recent studies have indicated that cytoskeletal proteins, $\mathrm{mdm} 2$, and p53 may be additional targets for this pathway: ${ }^{6}$ in the case of p53, neddylation inhibits transcriptional activity. ${ }^{6}$ Cullins are common subunits of Roc1/Rbx1/Hrt1-Skp1-Cullin/Cdc53-F box (SCF) complexes, which regulate the stability of various proteins involved in cell cycle control and signal transduction. ${ }^{7-10}$ Neddylation of cullins regulates the E3 ubiquitin ligase activity of Roc1/Rbx1/Hrt1-Skp1-Cullin/Cdc53-F box (SCF) complexes. Recent studies have shown that NEDD8 modification facilitates the recruitment of the E2 ubiquitinconjugating enzyme to the SCF complex and mediates the dissociation of $\mathrm{p} 120^{\mathrm{CAND} 1}$ an inhibitor of SCF ligase activity. ${ }^{11,12}$ In Drosophila NEDD8 mutants, there is an accumulation of protein levels for the cell cycle regulator, Cyclin $E$ (CycE), and for the signal transduction effectors, Cubitus interruptus ( $\mathrm{Ci}$ ), and Armadillo (Arm). ${ }^{13}$ Recently, NEDD8 has been shown to be incorporated into a variety of ubiquitinated inclusions, including the neurofibrillary tangles present in AD. ${ }^{14}$

APP is the precursor of the amyloid $\beta(\mathrm{A} \beta)$ peptide, the main component of senile plaques in AD. APP is synthesized as a transmembrane glycoprotein composed of extracellular and cytoplasmic domains. ${ }^{15,16}$ APP undergoes proteolytic cleavage with release of an ectodomain into the extracellular space. ${ }^{17-19}$ The APP homolog in Drosophila is called APP-like protein (APPL), ${ }^{15}$ a pan-neural protein belonging to the conserved APP family. Homology among APP members is paralleled by their ability to substitute for each other

\footnotetext{
${ }^{1}$ School of Biological Science, Seoul National University, Seoul, Korea; ${ }^{2}$ Department of Environmental Science, Policy and Management, Division of Insect Biology, Berkeley, CA, USA; ${ }^{3}$ Department of Molecular and Cell Biology, Division of Neurobiology, University of California, Berkeley, CA, USA and ${ }^{4}$ Hormone Research Center, School of Biological Sciences and Technology, Chonnam National University, Gwangju, Korea

${ }^{*}$ Corresponding author: J Yim, School of Biological Science, Seoul National University, Seoul 151-742, Korea. Tel: + 8228806702 ;

Fax: + 822871 4315; E-mail: jyim@snu.ac.kr

Keywords: Alzheimer's disease; apoptosis; cullin; NEDD8; SCF ligase

Abbreviations: AD, Alzheimer's disease; ap, apterous; APP, $\beta$-amyloid precursor protein; APP-BP1, $\beta$-amyloid precursor protein binding protein 1 ; APPL, APP-like protein; $\mathrm{Ci}$, cubitus interruptus; Da, daughterless; ECD, extracellular domain; EST, expressed sequence tag; ICD, intracellular domain; RT-PCR, polymerase chain reaction after reverse transcription of RNA; SDS-PAGE, sodium dodecyl sulfate polyacrylamide gel electrophoresis; SCF complexes, Roc1/Rbx1/Hrt1-Skp1-Cullin/ Cdc53-F box complexes; TUNEL, terminal deoxynucleotidyl transferase mediated-biotinylated UTP nick end labeling; UTR, untranslated regions

Received 25.8.05; revised 02.2.06; accepted 14.2.06; Edited by J Abrams; published online 21.4.06
} 
functionally: transgenes encoding either Drosophila Appl or human neural App will rescue the behavioral defects of Appl null flies. ${ }^{20}$ Different forms of Drosophila APPL protein are enriched in different areas of the brain. ${ }^{21}$ Deletion of the Appl gene does not cause obvious defects in adult brain morphology. ${ }^{20}$ However, loss- and gain-of-function studies have revealed a role for APPL in synapse differentiation and axonal transport. ${ }^{22-24}$ The mutant loechrig presents with a neurodegenerative phenotype that is strongly enhanced by App $^{d}$ mutants. ${ }^{25}$ Overexpression of wild-type and mutant APPL proteins has also been shown to cause abnormal neurite outgrowth. ${ }^{26}$ The cytoplasmic C-terminus of APP plays critical roles in cellular trafficking and delivery of proteases. Adaptor proteins with phosphotyrosine-binding (PTB) domains, including those in the X11, Fe65, and c-Jun $\mathrm{N}$-terminal kinase (JNK)-interacting protein (JIP) families, bind specifically to the highly conserved -YENPTY- motif in the APP C-terminus to regulate its trafficking, processing, and transcriptional modulation. ${ }^{27}$

To understand further the role of the Drosophila ortholog of APP-BP1 (dAPP-BP1), we have generated null mutations in dAPP-BP1. We have discovered that the loss of dAPP-BP1 blocks the NEDD8 conjugation pathway and causes apoptosis in imaginal disc cells. We find that dAPP-BP1 binds to the intracellular domain (ICD) of APPL, and dAPP-BP1 mutations rescue $A p p /^{d}$ phenotypes. Overexpression of APPL inhibits the NEDD8 conjugation pathway, disrupts the normal bristle pattern in the fly thorax, and induces apoptosis in wing imaginal discs. These APPL overexpression phenotypes are greatly enhanced by reduction in dAPP-BP1 level.

\section{Results}

dAPP-BP1 encodes the Drosophila homolog of APPBP1, a component of the neddylation pathway. The $d A P P-B P 1^{L P 67}$ mutant was identified in an enhancer-trap mutagenesis using the transposon $P\left(w^{+}\right.$lac $\left.Z\right)$ as mutagen. The screen selected for flies that were sluggish or died prematurely. Twenty mutant lines were identified: the $d A P P$ $B P 1^{L P 67}$ mutation was one causing a shortened lifespan. The $P$-element insertion site for $d A P P-B P 1^{L P 67}$ is located $79 \mathrm{bp}$ upstream of the translation initiation site, within the $5^{\prime}$-untranslated region (UTR) of CG7828. CG7828 is homologous to human APP-BP1 (Figure 1c) and was named $d A P P-B P 1$. Five cDNAs corresponding to dAPPBP1 from the Berkeley Drosophila Genome Project were AT09990, AT15603, LP03107, AT26180, and BE977705. Sequence analysis showed that the most complete cDNA, AT09990, consists of $1846 \mathrm{bp}$, encoding a putative protein of 524 amino acids, with $165 \mathrm{bp}$ of $5^{\prime}$ UTR and $59 \mathrm{bp}$ of $3^{\prime}$ UTR. The dAPP-BP1 gene spans about $2.3 \mathrm{~kb}$ of genomic DNA. The gene contains six exons spliced to yield a single $1846 \mathrm{bp}$ transcript, as determined by Northern blot analysis and cDNA sequencing (Figure 1b, data not shown). The deduced dAPP-BP1 shows canonical features present in human APP-BP1, including coiled coil motif and the UBA/THIFtype NAD/FAD-binding domain.

dAPP-BP1 is essential for Drosophila development. Two deletion mutants of $d A P P-B P 1$ were generated by imprecise

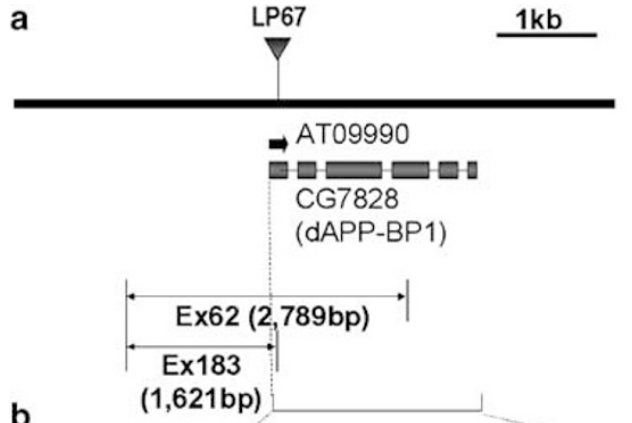

b

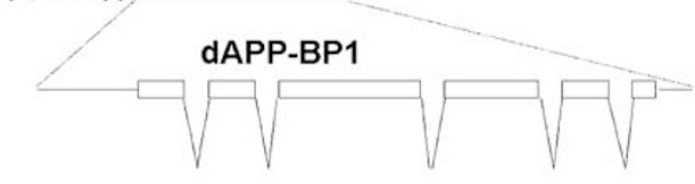

C

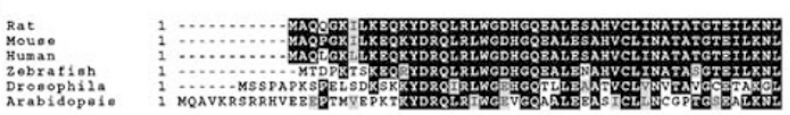

Rat
Mourae
Muman
Zobrat
Drogog

Human
Zobrafiah
Droogophila
Arabidopoia

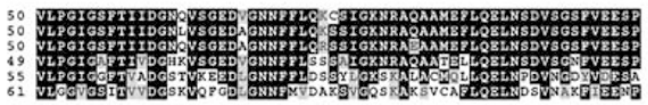

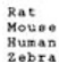

Ruman
zebratiob
Droophi1
Arabidopa

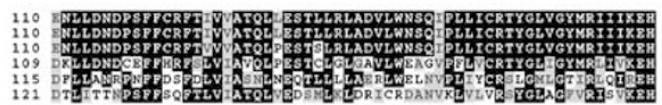

Rat
Moü
Muman
zebran
ebrat

2ebrafieh
Drooophils
Arabidopgia

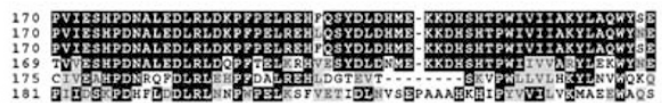

Rat
Mouga.
Muxan
Zebrat
ebrat

Ruman
Zetratish
Droogophila
Arabidope

Arabi

Rat
Mouga.
Kuman
2obra

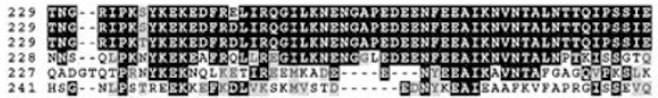

Ruman
Zubratio
Drono
Kagh

Droaphila
Arabidopei.

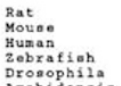

Droaophila
Arabidopai

Rat
Mouge
Muman

Moum
Munan
Zebrafiah

Dronophila
Arabidopoi.

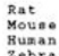

Ruzan
Zebrafiob
Droogoptos

Drosophila,
Arabidopois

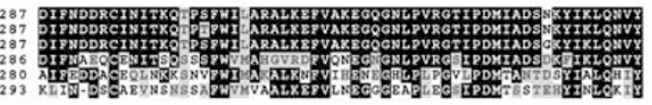

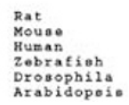

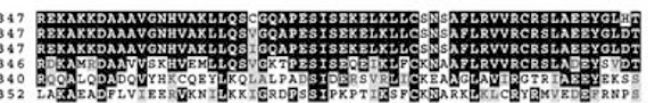

igure 1 Molecular map and sequence alignment of $d A P P-B P 1 . \quad(a)$ Transposon insertion sites are indicated above the map by triangles: $\triangle A P P$. $B P 1^{L P 67}$ is located about $200 \mathrm{bp}$ downstream of the transcription initiation site, within the $5^{\prime} U T R$ of $d A P P-B P 1$ mRNA. The dAPP-BP1 ${ }^{E \times 62}$ and $d A P P-B P 1^{E \times 183}$ lines were obtained by P-element excision of $d A P P-B P 1^{L P 67}$ and the excised regions are represented by horizontal arrows. In ${ } A P P-B P 1^{E \times 62}, 2789$ bp is deleted: the first three exons and $30 \mathrm{bp}$ of the fourth exon of dAPP-BP1. The dAPP-BP1 ${ }^{E x 183}$ mutation has $1621 \mathrm{bp}$ deleted. (b) Diagram of the $d A P P-B P 1$ transcript. The $2.3 \mathrm{~kb}$ region indicated by a bracket shown in (a) is enlarged. The $D A P P-B P 1$ gene has six exons and produces a $1846 \mathrm{bp}$ transcript. (c) Sequence comparison of dAPP-BP1: dAPP-BP1 shares $36-43 \%$ identity with other APP-BP1 proteins from Arabidopsis to mammals 
excision of the $d A P P-B P 1^{L P 67}$ transposon. In $d A P P-B P 1^{E x 62}$ flies, $2789 \mathrm{bp}$ was deleted, including the first three exons and 31 bases of the fourth exon of $d A P P-B P 1$. The $d A P P$ $B P 1^{E x 183}$ mutation is a $1621 \mathrm{bp}$ deletion including $84 \mathrm{bp}$ of $5^{\prime}$ UTR (Figure 1a). By polymerase chain reaction after reverse transcription of RNA (RT-PCR), we confirmed that $d A P P-B P 1^{E x 62}$ is a null mutant of dAPP-BP1 (Figure 2e). The $d A P P-B P 1^{E x 62}$ mutant is lethal in third instar larvae; lethality is rescued by overexpression of dAPP-BP1 (Supplementary Table S1). The temporal expression pattern of $d A P P-B P 1$, examined using quantitative RT-PCR (Figure 2f), showed expression at all developmental stages.

Loss-of-function phenotypes for $\triangle A P P-B P 1$ mutations were examined by mosaic analysis. For $d A P P-B P 1^{E x 62}$ very few mutant cells could be found in adult eyes (Figure $2 b$ ). For dAPP-BP1 ${ }^{E x 183}$ mosaics, however, mutant and wild-type clones were frequently observed (Figure 2a). Mutant clones were substantially smaller than wild type, suggesting that mutant cells were defective in proliferation or survival. Consistent with this interpretation, third instar larvae for $d A P P-B P 1^{E x 62}$ homozygote and $d A P P-B P 1^{E x 62} / D f(3 L) / x d 6$ genotypes have unusually small imaginal discs (Figure $2 d$ ). Taken together, these observations indicate that dAPP-BP1 is essential for normal tissue development in Drosophila.

The $d A P P-B P 1$ mutation blocks the neddylation pathway. The APP-BP1 and UBA3 heterodimer has been reported to be responsible for E1-like functions in NEDD8 activation. $^{2}$ If dAPP-BP1 participates in neddylation, mutant phenotypes would be expected to be similar to those of NEDD8 mutants. NEDD8 modification is necessary for $\mathrm{SCF}^{\text {slimb }}$-mediated protein degradation in Drosophila wing discs. ${ }^{13}$ Thus, protein stability was examined for substrates of Slimb in $d A P P-B P 1$ mutant cells. In developing wing discs, $\triangle A P P-B P 1$ mutant cells accumulated high levels of full-length $\mathrm{Ci}\left(\mathrm{Ci}^{\mathrm{FL}}\right)$ and Arm proteins (Figure $3 \mathrm{j}-\mathrm{l}$ and $\mathrm{m}-\mathrm{O}$ ). These are similar to accumulations previously reported for NEDD8 mutants. $^{13}$
In eye discs, $\mathrm{Ci}^{\mathrm{FL}}$ accumulated in $\triangle A P P-B P 1$ clones located anterior to the morphogenic furrow (MF) (Figure 3d-f, arrows). This is similar to $\mathrm{Ci}^{\mathrm{FL}}$ accumulation observed in MF cells that transduce the $\mathrm{Hh}$ signaling pathway. $\mathrm{Ci}^{\mathrm{FL}}$ accumulation was also observed in some posterior mutant cells. Similar to accumulation previously described for NEDD8 mutant clones, ${ }^{13}$ the $\mathrm{Ci}^{\mathrm{FL}}$ accumulation observed here was in cells located proximal to the MF (Figure $3 d-f$, arrowheads), but not in cells located distal to it (Figure $3 d-f$, asterisks). Eye discs were also examined for accumulation of Cul1, since previous investigations ${ }^{13}$ have shown that neddylation controls Cul1 stability. Cul1 has been found to accumulate in NEDD8 mutant clones. Here, Cul1 was found to accumulate in $d A P P$ $B P 1$ null clones (Figure $3 \mathrm{~g}-\mathrm{i})$. Clones of $d A P P-B P 1^{E \times 183}$ resembled wild-type clones (Figure $1 \mathrm{a}-\mathrm{c}$ and data not shown). Neddylated forms of Cul1 are missing and unneddylated Cul1 is greatly accumulated in cell extracts from eye discs and larval brain (Figure 3p). Taken together, all of these observations appear to be due to loss of $d A P P-B P 1$, with the indication that dAPP-BP1 is an essential component of the Drosophila neddylation pathway.

The loss of $d A P P-B P 1$ causes apoptosis, not cell proliferation. Growth properties of $d A P P-B P 1$ mutant cells were examined by comparing marked pairs (twin spots) of clones in the wing disc. Homozygous $d A P P-B P 1^{E x 62}$ mutant clones were found to be substantially smaller than their wildtype twins, by about a factor of three (Figure $4 \mathrm{~s}$ and $\mathrm{t}$ ). In addition, the $d A P P-B P 1^{E x 62}$ clones completely lacked elavstaining, indicating the absence of differentiated neurons. Interestingly, homozygous $d A P P-B P 1^{E x 183}$ mutant clones were found to be similar in size to their wild-type twins (Figure $4 q$ and $r$ ). These observations indicate that the absence of dAPP-BP1 protein causes a decrease in growth during development.

Two possible mechanisms were considered, and experiments designed to distinguish between them. The first mechanism is that the absence of dAPP-BP1 could reduce growth via decreased cell proliferation. Alternatively, the
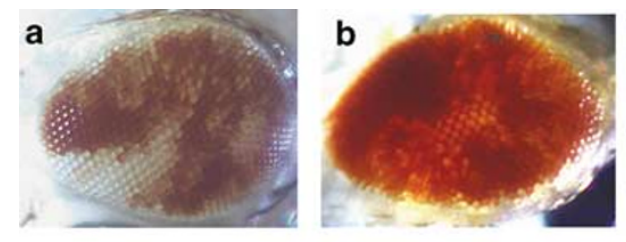

e

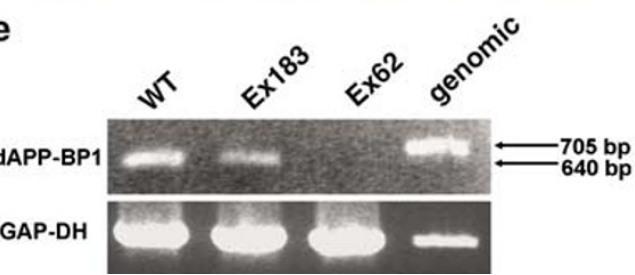

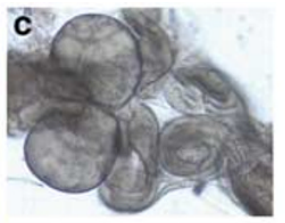

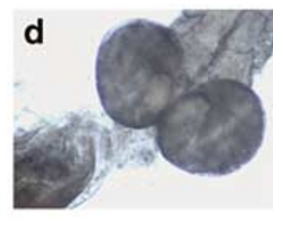

f

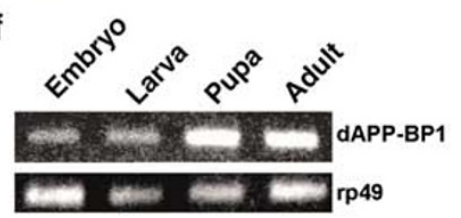

Figure $2 d A P P-B P 1$ mutant phenotypes and developmental expression. (a) The adult eyes of $w$ ey-flp;; $P\{U b i-G F P\} 61 E F P\{n e o F R T\} 80 B / d A P P-B P 1{ }^{E x 183} P\{n e o F R T\} 80 B$. (b) The adult eyes of $w$ ey-flp;; P\{Ubi-GFP\}61EF P\{neoFRT\}80B/dAPP-BP ${ }^{E \times 62} P\{$ neoFRT $\} 80 B$. The $d A P P-B P 1^{E x 183}$ clones containing white ommatidia exist in (a), compared to (b) in which $d A P P-B P 1^{E \times 62}$ clones (marked by white ommatidia) are absent, leaving a defective eye that contains $d A P P-B P 1^{E \times 62} /+$ or $+I+$ cells in red ommatidia. (c) Wild-type brain and eye imaginal disc. (d) $d A P P-B P 1^{E x 62} / D f(3 L) / x d 6$ eye discs are almost demolished. (e) mRNA expression analysis in third instar larvae (RT-PCR): $d A P P$ $B P 1$ mRNA is not expressed in $A A P P-B P 1{ }^{E \times 62}$ homozygotes. (f) Quantitative RT-PCR analysis of $d A P P-B P 1$ expression during development. As control, transcripts of $r p 49$ were amplified. $d A P P-B P 1$ transcripts can be detected during all developmental stages 
absence of dAPP-BP1 could trigger apoptosis that would reduce growth through the removal of neuronal cells. Apoptosis was examined in the eye discs of $d A P P-B P 1^{E x 62}$ homozygotes. In normal eye discs, apoptosis is very low, as indicated by terminal deoxynucleotidyl transferase mediatedbiotinylated UTP nick end labeling (TUNEL) markers and the cleaved (active) form of caspase 3 (Figure $4 b$ and d). In contrast, there are massive levels of caspase 3 (Figure $4 \mathrm{c}$ )
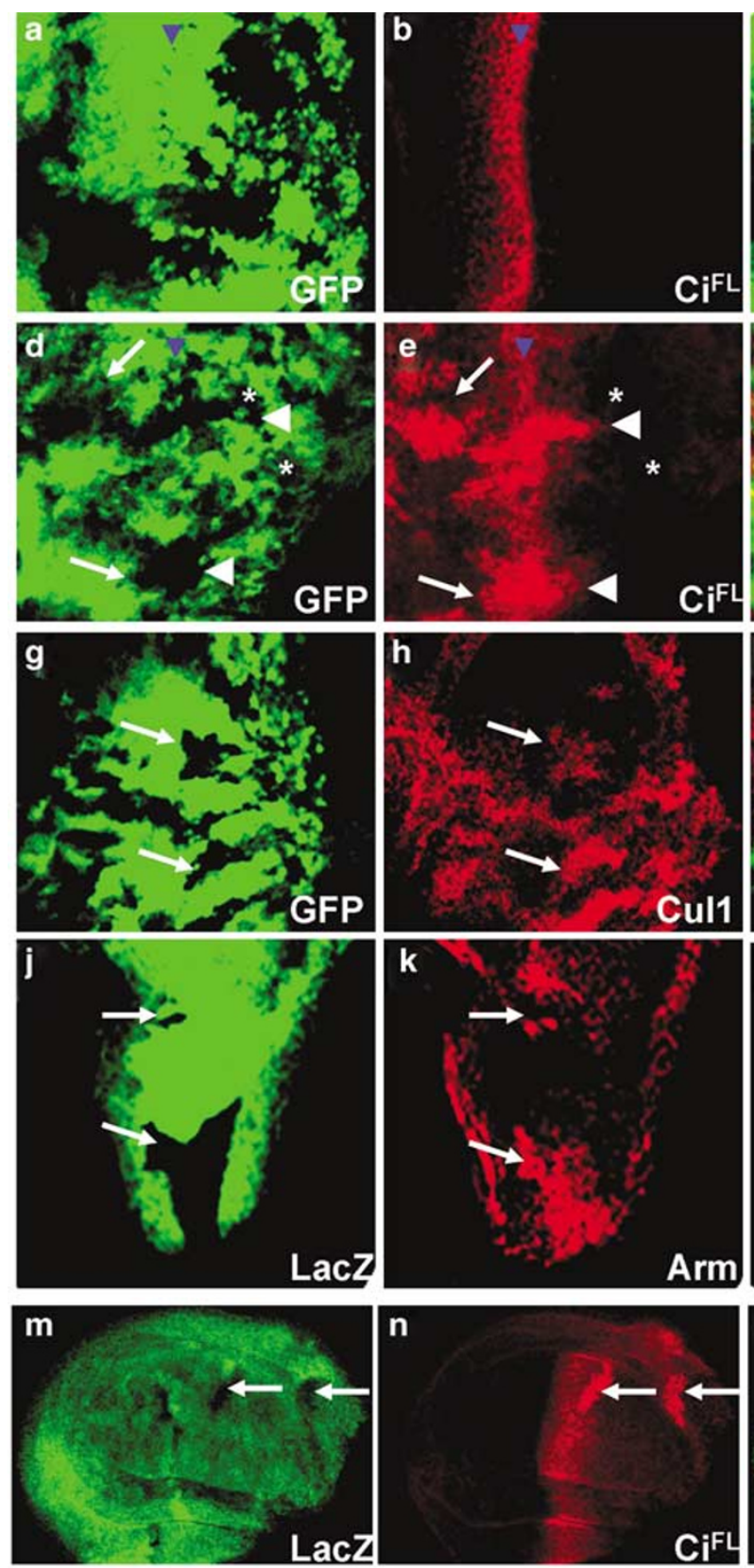

p

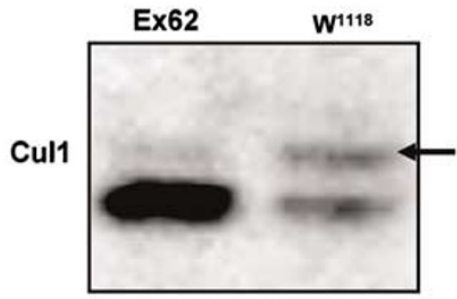

B-Tubulin
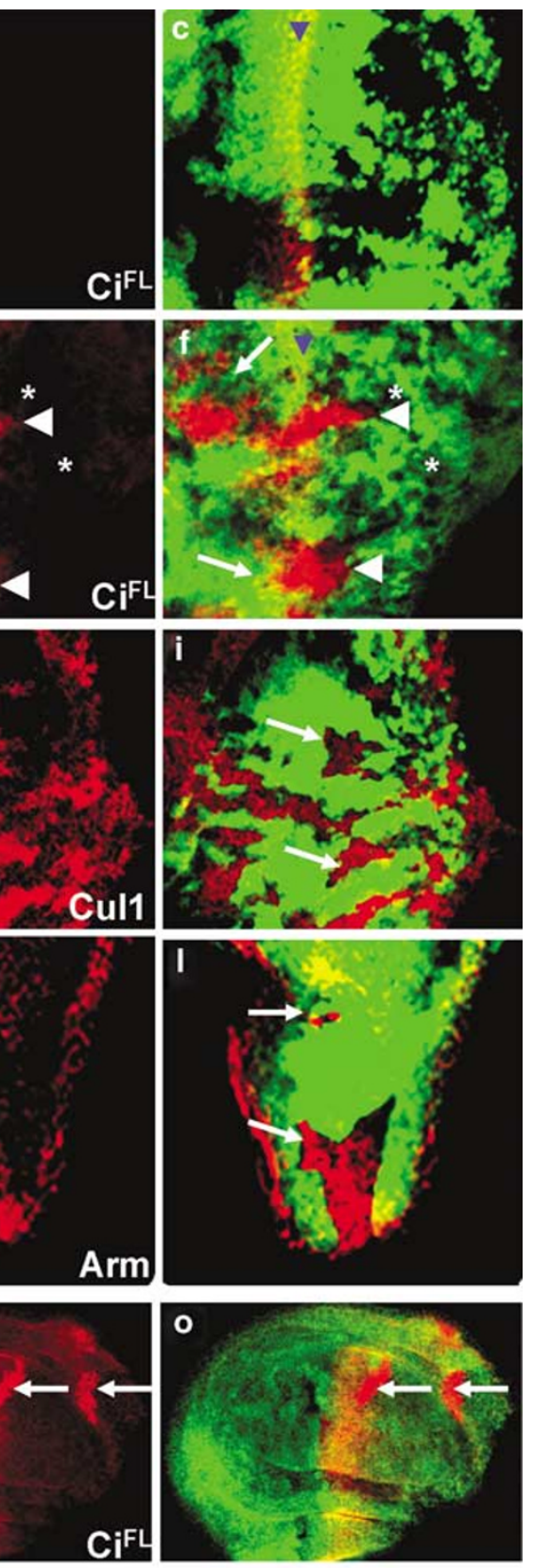
and TUNEL signal (Figure 4a) observed all across the eye discs of $d A P P-B P 1$ mutants. Caspase 3 is also increased in $d A P P-B P 1^{E x 62}$ clones in wing discs and in eye discs (Figure
$4 \mathrm{e}-\mathrm{j})$. The rate of cell division in dAPP-BP1 mutant clones was examined using an antibody to the phosphorylated form of histone 3 (PH3). PH3 staining is uniform over eye discs
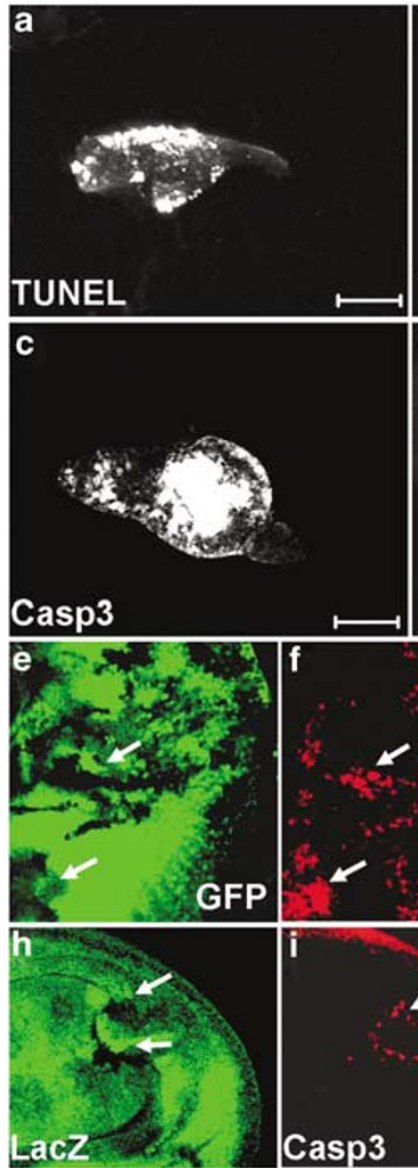

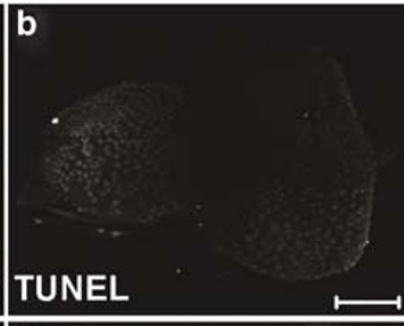

Tu
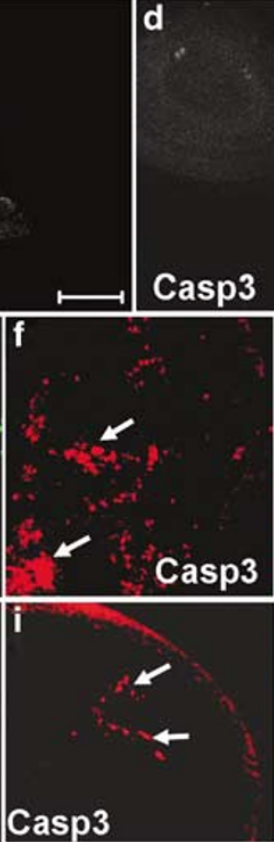
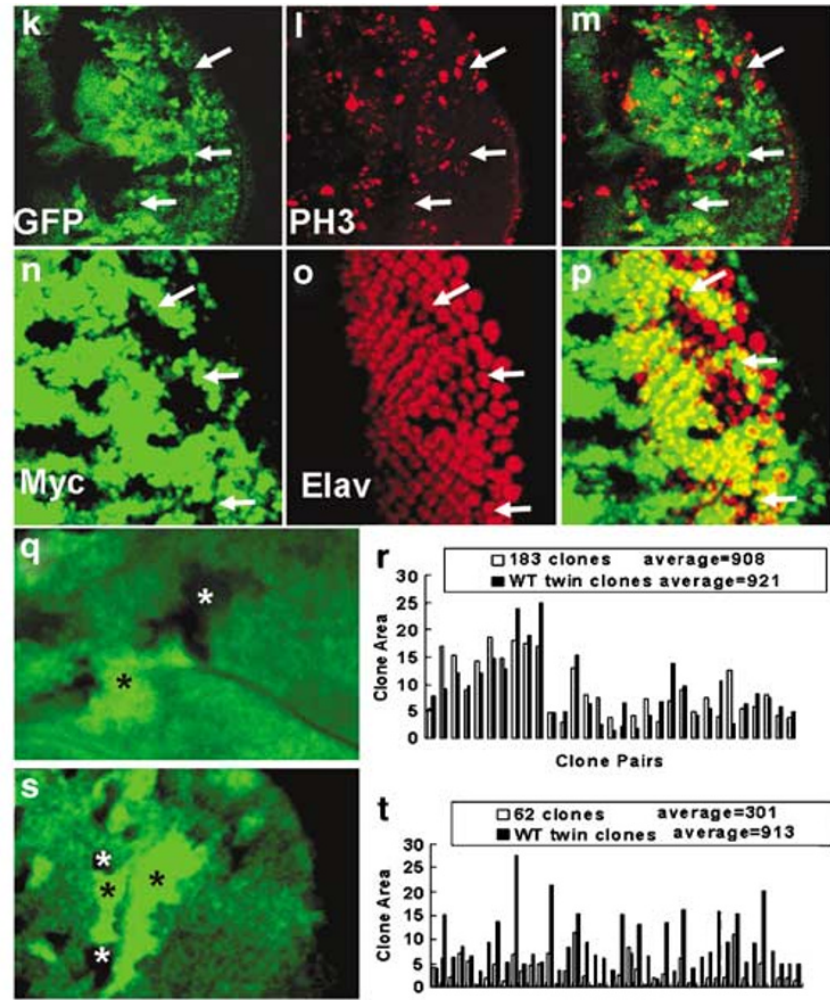

Clone Pairs

Figure 4 The loss of dAPP-BP1 causes apoptosis, not cell proliferation. (a-d) Third instar eye discs. Scale bar is $50 \mu \mathrm{m}$. (a, b) TUNEL assay. White indicates TUNEL signals. (c, d) Immunostaining by anti-activated caspase 3 . (Casp3 = anti activated caspase 3). White indicates anti-activated caspase 3 positive site. (a) $d A P P-B P 1{ }^{E x 62}$ homozygote. The size of eye disc is greatly reduced and TUNEL signals are greatly increased compared with the $w^{1118}$ wildtype eye disc (b). (c) $d A P P-B P 1^{E \times 62}$ homozygote. The level of activated caspase 3 is greatly increased in the wildtype, $w^{1118}(\mathbf{d})$. Clones carrying $d A P P-B P 1^{E \times 62}$ are marked by the lack of GFP $(\mathbf{e}, \mathbf{g}, \mathbf{k}, \mathbf{m})$ or beta-galactosidase $(\mathbf{h}, \mathbf{j}, \mathbf{k}, \mathbf{m})$ expression. (e-g) The level of activated caspase is increased in $d A P P-B P{ }^{E x 62}$ clones in 3rd larvae eye disc (arrows). (h-j) The amount of activated caspase is increased in mutant clones in 3rd larvae wing disc (arrows). (k-m) PH3 is anti -phospho histone H3. $d A P P-B P 1^{E x 62}$ cells retain the ability to proliferate. (n-p) Clones carrying $\triangle A P P-B P 1^{E \times 62}$ are marked by the lack of myc expression. The number of elav-positive cells is decreased in $d A P P-B P 1^{E \times 62}$ clones (arrows) (q) $d A P P-B P 1^{E \times 183}$ homozygous clones and twin spots (white asterisk $=d A P P-B P 1^{E x 183}$ clones (arrows) homozygous clones were marked by lack of $\beta$-galactosidase expression. Black asterisk $=$ twin spots. $\beta$-galactosidase = Green). (r) Area measurements of 31 pairs of homozygous $d A P P-B P 1^{E x 183}$ mutant and wild-type twin clones (scale: 100 pixels). Wild-type and mutant cells are produced in the same cell division, so differences in clone area reflect differences in growth or cell survival after clone induction. (s) $d A P P$-BP $1^{\text {Ex62 }}$ homozygous clones and twin spots (white asterisk $=d A P P-B P 1^{E x 62}$ homozygous clones were marked by lack of $\beta$-galactosidase expression. Black asterisk $=$ twin spots. $\beta$-galactosidase $=$ Green). (t) Area measurements of 42 pairs of homozygous $d A P P-B P 1^{E x 62}$ mutant and wild-type twin spots (scale: 100 pixels). The area of wild-type twin clones is about three times larger than that of mutant clones

Figure $3 d A P P-B P 1$ mutation blocks NEDD8 conjugation pathway. Accumulation of $\mathrm{Ci}^{\mathrm{FL}}$, Armadillo, and Cul1 in $d A P P-B P 1$ mutant cells. (a-c) Clones carrying $d A P P$ $B P 1^{E x 183}$ are marked by the lack of GFP expression $(\mathbf{a}, \mathbf{c})$. (a-i) The blue arrowheads mark the position of the morphogenetic furrow (MF) in this and following figures. For imaginal discs, anterior is to the left. (d-l) Clones carrying $d A P P-B P 1^{E x 62}$ are marked by the lack of GFP (d, g) or beta-galactosidase (j, $\left.\mathbf{m}\right)$ expression. (d-f) A late third-instar eye disc containing $A A P P-B P 1^{E \times 62}$ mutant clones and stained by anti-Ci ${ }^{\mathrm{FL}}$ antibody. In mutant cells, $\mathrm{Ci}^{\mathrm{FL}}$ protein levels (arrows) are similar in the MF and in the cells anterior to it. In posterior cells, $\mathrm{Ci}^{\mathrm{FL}}$ persists, albeit at a lower level (arrowheads), and is not detected in the most posterior cells (asterisks). This pattern is similar to those observed in NEDD8 null clones ${ }^{13}$ (g-i) A late third-instar eye disc containing $d A P P-B P 1^{E x 62}$ mutant clones and stained with anti-Cul1 antibodies (red). Cul1 accumulates in $d A P P-B P 1^{E x 62}$ mutant clones (arrows) that are marked by a lack of GFP expression (green in $\mathbf{g}, \mathbf{i})$. (j-I) Accumulation of Armadillo observed in $d A P P-B P 1^{E \times 62}$ mutant clones in the wing disc (arrows). ( $\mathbf{m}-\mathbf{0})$ Accumulation of $\mathrm{Ci}^{\mathrm{FL}}$ observed in the $d A P P-B P 1^{E x 62}$ mutant clones in wig disc (arrows). (p) Ex62 is $d A P P-B P 1^{\text {Ex62 }}$ homozygote cell extracts. Western blot analysis of cell extracts from third-instar eye discs and brain lobes by anti-Cul1 antibodies indicates that the Cul1 protein is accumulated and the neddylated form of Cul1 (arrow) is almost missing in the $d A P P-B P 1^{E \times 62}$ homozygote 
containing $d A P P-B P 1^{E x 62}$ clones, indicating that $d A P P-B P 1$ null cells retain the ability to proliferate. Thus, these observations are consistent with a role for dAPP-BP1 in either the regulation of apoptosis or in the regulation of developmental pathways that, when improperly controlled, lead to apoptosis.

The dAPP-BP1 protein interacts with the amyloid precursor protein-like protein. Original identification of APP-BP1 was based on its binding with the ICD of APP. ${ }^{1}$ The binding site has been identified as the carboxyl-terminal 31 amino-acid residues of APP. ${ }^{4}$ This suggests the possibility of a similar interaction between dAPP-BP1 and APPL. Interaction was examined by an in vitro binding assay using a bacterially expressed GST-tagged APPLC63 (APPL carboxyl-terminal 63 amino-acid residues), GST, and in vitro expressed ${ }^{35} \mathrm{~S}$-methionine-labeled dAPP-BP1. Using this assay, dAPP-BP1 protein is detected only in GST-APPLC63 pre-adsorbed glutathione-agarose beads (Figure 5f). Thus, similar to the described APP-BP1 interaction with $A P P$, interaction is observed here between dAPP-BP1 and APPL via the APPL ICD.

Interaction between APPL and dAPP-BP1 was examined further by Western blot using anti-APPL antibody. This analysis shows that the level of APPL protein is correlated with the overall amount of dAPP-BP1. Cell extracts from a genetic background where dAPP-BP1 is overexpressed show that APPL levels are greatly increased (about six-fold)

a

Percentage of Normal Scutellar Macrochaete $(n=100)$

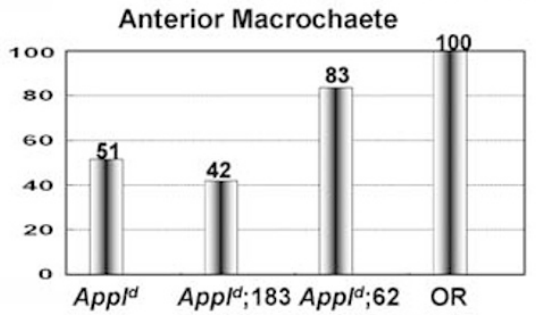

Posterior macrochaete

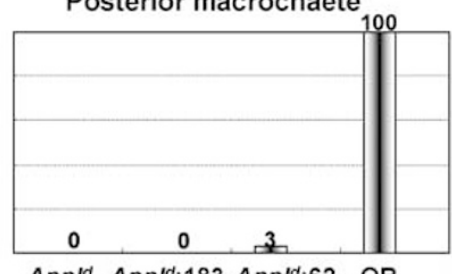

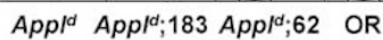

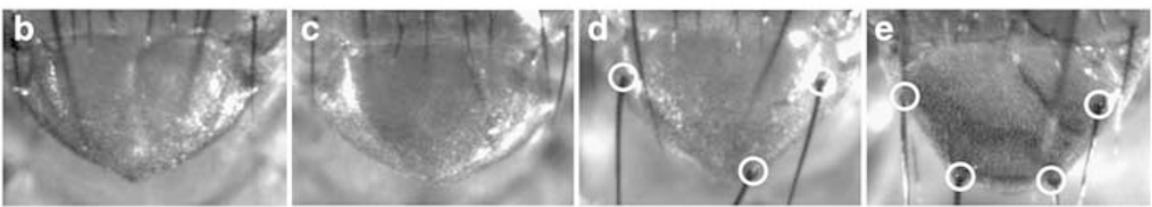

f

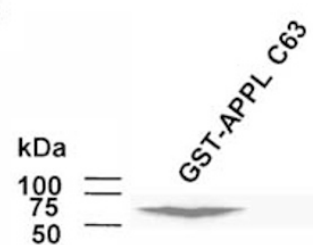

$\mathrm{g}_{A p p / 4} \frac{\text { Da-gal4 }}{\text { UAS-dAPPBP } 1} w^{1118}$ dAPP-BP1 text
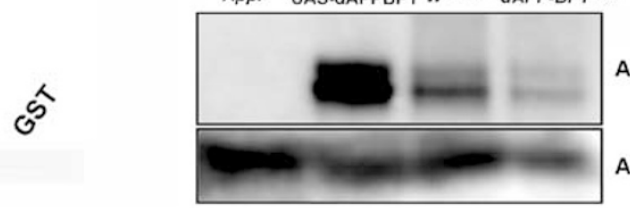

Anti-APPL

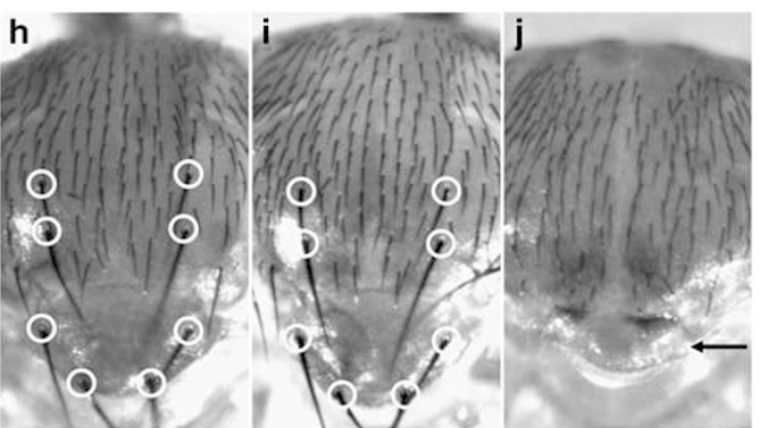

$\mathbf{k}$

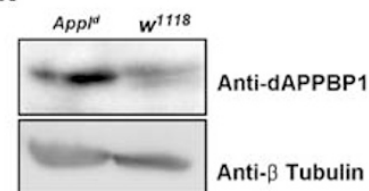

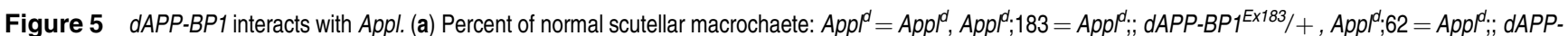
$B P 1^{E \times 62} /+, \mathrm{OR}=$ Oregon $R$. Missing macrochaete phenotype of $A p p l^{d}$ flies is suppressed by $d A P P-B P 1^{E \times 62}$. (a-e) All of these flies were incubated at $25^{\circ} \mathrm{C}$. (b-e) Fly scutellum. White circle indicates macrochaete. (b) $A p p^{d}$, (c) $A p p{ }^{d}$;; $d A P P-B P 1^{E x 183} /+$, (d) $A p p f^{d}$;; $d A P P-B P 1^{E \times 62} /+$, and (e) Oregon $R$. (f) Binding studies performed with the bacterially expressed GST-tagged APPL C63 (APPL carboxy-terminal 63 amino acids), GST, and the in vitro-expressed dAPP-BP1. GST-APPL C63 specifically binds with dAPP-BP1. (g) Western blot analysis of cell extract from third-instar eye discs and brain lobes by anti-APPL antibodies. Loss of dAPP-BP1 slightly decreases APPL protein levels, but overexpression of dAPP-BP1 greatly increases the amount of APPL proteins. Da-GAL4 is expressed in all imaginal discs. (h-j) Adult thorax of flies incubated at $29^{\circ} \mathrm{C}$, the temperature at which GAL4 induction is most efficient. White circle indicates macrochaete. (h) ap-GAL4/ +, (i) UAS-dAPPBP1/ +, (j) ap-GAL4/ +; UAS$\triangle A P P B P 1 /+$. In the dAPP-BP1 overexpression condition, the size of the scutellum (arrows) is decreased and most macrochaetes are missing. (k) Western blot using an antidAPPBP1 antiserum. The amount of dAPP-BP1 is increased in $\mathrm{Appl}^{d}$ adult heads 
compared to wild-type controls. In a $d A P P-B P 1^{E x 62}$ mutant background, APPL levels are greatly reduced compared to wild-type controls.

The $d A P P-B P 1$ mutation partially suppresses the thorax phenotype of $\boldsymbol{A p p l}^{\boldsymbol{d}}$. In addition to physical interactions between dAPP-BP1 and APPL proteins, genetic interactions are observed between $\triangle A P P-B P 1$ and $A P P L^{d}$ mutations. Previously, Appl mutations have been found to cause macrochaete deletions in the scutellar region of the adult thorax. ${ }^{28}$ The $A p p{ }^{d}$ macrochaete-missing phenotype is due to the deletion of external cells and includes the absence of macrochaete-associated neurons. ${ }^{28} A p p{ }^{d}$ flies that are also heterozygous for $d A P P-B P 1^{E x 62}$ show rescue of the macrochaete (Figure 5a-e). In the $d A P P-B P 1^{E x 62}$ heterozygotes, approximately $30 \%$ of the missing anterior scutellar bristles are restored to the $A p p /^{d}$ mutants (Figure $5 \mathrm{a}$ ). Thus, $d A P P-B P$ loss of function acts as a suppressor of $A p p / d$. Overexpression of dAPP-BP1 also shows macrochaete-missing phenotype, and the protein level of dAPP-BP1 is increased when APPL is absent (Figure 5j and $\mathrm{k}$ and Supplementary Figure S1). From these observations, the suggestion is that $A p p /^{d}$ mutant phenotypes are caused by abnormal accumulation of dAPP. BP1. The excess quantity of dAPP-BP1 can lead to low levels of apoptosis with loss of APPL-induced macrochaete elimination due to cell death (Figure $7 \mathrm{t}$ ).

APPL-induced toxicity is enhanced by $d A P P-B P 1$ mutation. Genetic interactions were investigated using an App/ transgene driven by apterous (ap)-GAL4. For flies in the ap-App/ strain, thorax phenotypes resembled those observed in wild-type flies (Figure 6a, first panel). Loss of one dose of the $d A P P-B P 1$ gene in an ap-Appl background, however, caused loss of thoracic macrochaetes and a reduction in scutellem size (Figure 6a, second panel, arrows). This phenotype resembles that seen in animals with two-fold Appl overexpression (Figure $6 \mathrm{a}$, third panel, arrows). The $d A P P-B P 1$ interaction with ap-Appl is sensitive to temperature. When reared at $29^{\circ} \mathrm{C}$, flies that are haplodeficient for $d A P P-B P 1$ in an ap-Appl background show an extreme phenotype: the loss of thoracic macrochaetes is massive and the scutellar size reduction is severe (Figure 6c).

Genetic interactions between ap-Appl and Cul1 indicate that reduction in Cul1 level enhances ap-Appl thorax phenotypes (Figure 6a, fourth panel, arrows). This suggests that the neddylation pathway plays a role in the Appl-induced phenotypes observed in the thorax: neddylation is an essential feature of Cul1 functions. If these phenotypes are caused by apoptosis, overexpression of baculoviral P35 protein should rescue APPL-induced thorax phenotypes. Baculoviral P35 protein inhibits caspases, thereby blocking apoptosis in many species. Figure $6 \mathrm{~d}$ shows that $\mathrm{P} 35$ rescues APPL-induced thorax phenotypes. The results of TUNEL and activated caspase 3 antibody staining in wing discs (Figure $6 \mathrm{e}-\mathrm{i})$ showed that APPL overexpression promoted apoptosis and this is enhanced by reduction of dAPP-BP1. The graph of Figure 6j confirms these results.
dAPP-BP1 overexpression suppresses $A P P L^{\text {sd }}$-induced apoptosis via the ICD of APPL. Ectopic expression phenotypes of APPL were examined using a GAL4 transgene to drive overexpression in the wing disc. MS1096 is an X-linked transgene that expresses GAL4 strongly on the dorsal surface of the wing primordium in third instar larvae; weak expression is observed on the ventral surface. Owing to dosage compensation, GAL4 levels in males (MS1096/ $Y$ ) are twice those in heterozygous females $(M S 1096 /+)$. $\mathrm{APPL}^{\text {sd }}$ and $\mathrm{APPL}^{\text {sd } \Delta \mathrm{IC}}$ overexpression caused a great reduction in wing size for males (Figure $7 \mathrm{e}$ and $\mathrm{g}$ ). Overexpression of $\mathrm{APPL}^{\mathrm{s}}$ and dAPP-BP1 caused minimal changes in the size of wings; wing size (Figure $7 \mathrm{~b}$ and $c$ ) is nearly the same as for control flies (Figure 7a). In the case of APPL, one copy of Appl (Figure 7d) failed to show significantly different phenotypes. Increasing expression further using two copies of the UAS- Appl transgene reduced wing size and caused the loss of cross-veins (Figure $7 \mathrm{i}$ and $\mathrm{j}$ ). The loss of cross-vein phenotype is rescued by co-expression of P35 (Figure $7 \mathrm{~m}$ ).

The reduction in wing size in APPL and its overexpression mutant forms could either be due to smaller cells or fewer cells. Every cell on the wing blade has a hair, so hair density marks cell density, and hence cell size. Wing hair density was unchanged on all the various APPL forms (data not shown). Therefore, in APPL and its mutant forms, overexpression causes a reduction in cell number responsible for the reduction of wing size. Apoptosis in wing imaginal discs was examined by TUNEL assay and immunostaining by antiactivated caspase 3 antibody. These experiments indicated a large increase in cell death throughout the wing pouch when $\mathrm{APPL}^{\text {sd }}$ and $\mathrm{APPL}^{\mathrm{sd} \Delta \mathrm{IC}}$ were overexpressed (Figure $7 \mathrm{p}$, $r$ and u). To test whether overexpression was sensitive to the dosage of dAPP-BP1, it was co-expressed with the APPL mutant forms. Only APPL $^{\text {sd }}$-induced apoptosis and smallwing phenotypes were rescued by dAPP-BP1 overexpression (Figure 7f, h, q, s and v). When dAPP-BP1 was overexpressed alone, low levels of apoptosis occurred (Figure 7t). These data suggest that APPL-induced toxicity is attenuated by dAPP-BP1 via the APPL ICD.

Overexpression of APPL and its derivatives inhibits SCF ligase function. The $\mathrm{Cul1} 1^{\mathrm{Ex}}$ null mutation ${ }^{13}$ was used to examine interaction between APPL and SCF ligase. Hériché et al. $^{29}$ reported that reduction of Cul1-mediated SCF function causes apoptosis. Here we show that reduction of Cul1 level enhances Appl-induced small wing and missing crossvein phenotypes (Figure $7 \mathrm{k}-\mathrm{m}$ ). Similarly, these phenotypes are enhanced by increasing the level of Appl (Figure 7i, j and $\mathrm{m}$ ). To assess Cul1 function directly, we sought a target protein whose destruction might be limited to the Cul1-SCF/proteasome pathway. Ci levels are particularly sensitive to activity in the SCF/proteasome pathway. The level of $\mathrm{Ci}$ processing can be scored by measuring the accumulation of C-terminal epitope. ${ }^{29}$ This assay showed that reduction of Slimb and Roc1, that contribute to SCF function, is associated with increased $\mathrm{Ci}$ staining. Here, reduction of Cul1 function by overexpressing APPL and its mutant forms (Figure $8 \mathrm{a}-\mathrm{d}$ ) led to $\mathrm{Ci}$ accumulation. These 
results indicate that $\mathrm{APPL}$ and its overexpression mutant forms reduce Cul1-mediated SCF function.

To test if inhibition is related to neddylation, Western blot analysis was performed with anti-Cul1 antibody. APPL and its derivatives were overexpressed by $A p p l-G A L 4$ at $29^{\circ} \mathrm{C}$. Cell extracts from third instar larval brain and eye discs were used for Western blots. Neddylated Cul1 is decreased in these cell extracts, with especially significant reductions observed for $A P P L^{s d}$. These results demonstrate that reduction of SCF function is due to inhibition of neddylation. Although $d A P P$ -
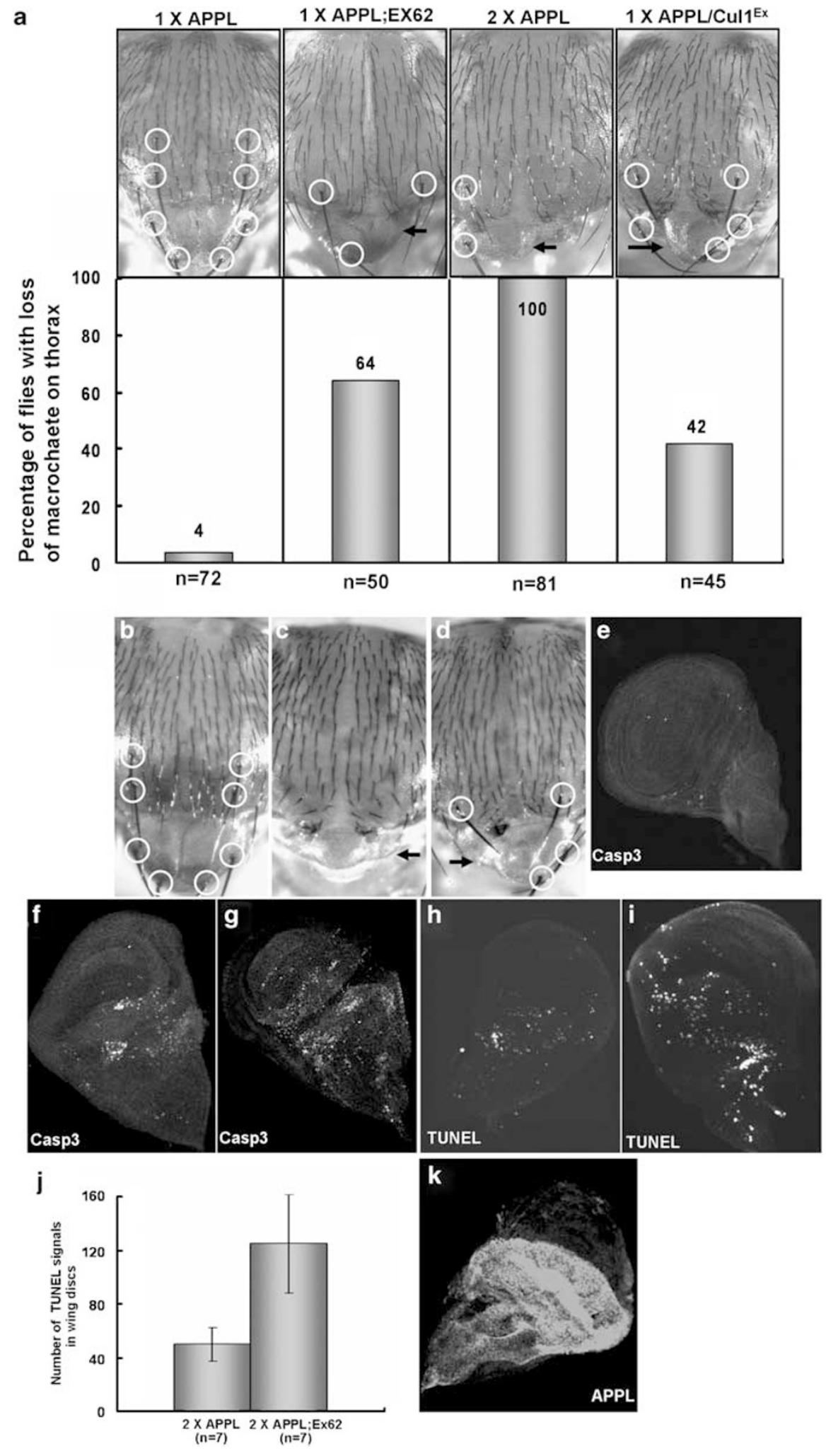

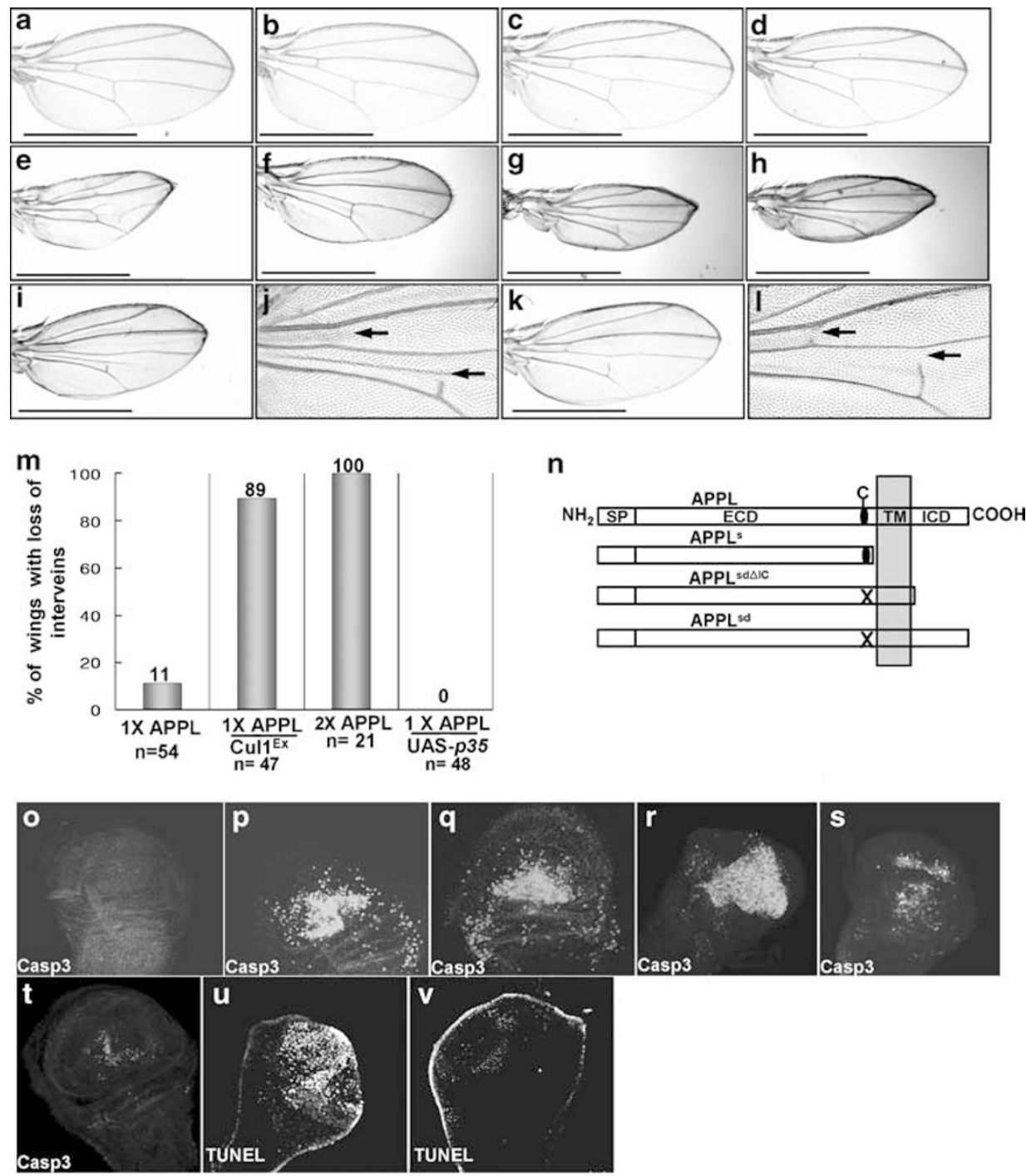

Figure 7 dAPP-BP1 overexpression rescues APPL ${ }^{\text {sd }}$-induced apoptosis. (a-h) Adult wing of Drosophila. Scale bar is $1 \mathrm{~mm}$. (a-m) The flies were raised at $25^{\circ} \mathrm{C}$. (a) Wild-type wing expressing only the GAL4 driver (MS1096/M). (b) MS1096/Y;UAS-dAPPBP1/+ . Same as (a). (c) MS1096/Y;UAS-AppF/+ . No difference compared to (a). (d) MS1096/Y;UAS-Appl/

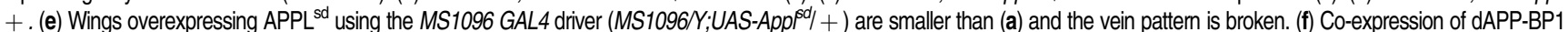
(MS1096/Y;UAS-App $\left.{ }^{\beta d} / U A S-d A P P B P 1\right)$ rescues wing phenotype of (e). (g) Wings overexpressing APPL ${ }^{\text {sd } \triangle I C}$ using the MS1096 GAL4 driver (MS1096/Y;UAS-App ${ }^{\beta d \Delta I C /}+$ ) are smaller than (a) and vein pattern is broken. (h) MS1096/Y;UAS-App ${ }^{\beta d A C} /$ UAS-dAPPBP1 similar to (g). (i) MS1096/Y;UAS-App/UAS-Appl. Increased level of APPL causes small wing and crossvein missing phenotypes (arrows). (j) Magnification of (i). Arrows indicate missing cross-vein. (k) Reduced level of Cul1 (MS1096/Y;UAS-Appl/Cul1 EX) enhances APPL overexpression phenotypes. (I) Magnification of (k). Arrows indicate missing cross-vein. (m) Quantification of the missing inter-vein phenotypes. P35 suppress APPL-overexpression pheotypes

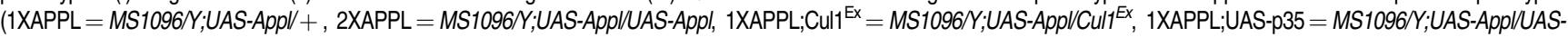
p35). (n) Schematic overview of mutated APPL molecules used. $E C D=$ extracellular domain, $\mathrm{TM}=$ transmembrane, $I C D=$ intracellular domain, $S P=$ signaling peptide, $C=$ cleavage

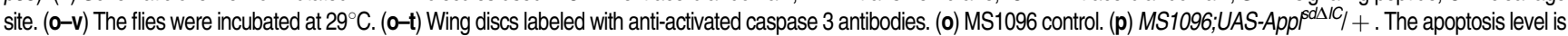

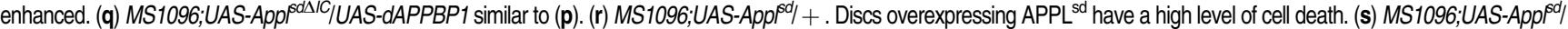
UAS-dAPPBP1. dAPP-BP1 overexpression rescues APPL ${ }^{\mathrm{sd}}$-induced apoptosis. (t) MS1096; UAS-dAPPBP1/UAS-dAPPBP1. dAPP-BP1 overexpression induce low levels of apoptosis.

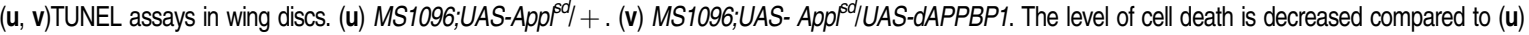

Figure 6 APPL-induced toxicity is enhanced by $d A P P-B P 1$ mutation. (a) Quantification of the phenotypes in the adult thorax for different genetic backgrounds. 1XAPPL =ap-GAL4,UAS-Appl/CyO; 1XAPPL;EX62 =ap-GAL4,UAS-App//CyO; dAPP-BP1 Ex62/+; 2XAPPL =ap-GAL4,UAS-Appl/UAS-Appl; 1XAPPL;Cul1 Ex $=a p-$ GAL4,UAS- Appl/Cul1 ${ }^{E x}$. Cul1 ${ }^{E x}$ is a deletion mutant of the Cul1 gene. In some genetic backgrounds, the size of scutellum (arrows) is decreased. These flies were raised at $25^{\circ} \mathrm{C}$. (a-d) White circle indicates macrochaete. (b-d) The adult nota of flies raised at $29^{\circ} \mathrm{C}$. (b) ap-GAL4,UAS-Appl. (c) ap-GAL4,UAS-Appl; dAPP-BP1 ${ }^{E \times 62}$. Almost all macrochaetes are missing, and the size of the scutellum is highly reduced (arrows). (d) ap-GAL4,UAS-Appl/UAS-p35; dAPP-BP1 Ex62/ + . (c) Phenotypes are partially rescued. Macrochaetes are restored and the size of the scutellum is almost completely rescued. (e-g) A wing disc stained for activated caspase 3 (white). The larvae were raised at $29^{\circ} \mathrm{C}$. (e) ap-GAL4; dAPP-BP1 ${ }^{E \times 62} /$. . (f) ap GAL4,UAS-Appl/UAS-Appl. Signals are increased over those seen in (e). (g) ap GAL4,UAS-Appl/UAS-Appl; dAPP$B P 1^{E \times 62} /+$. The activated caspase-positive cells are increased compared with (f). (h, i) TUNEL assay in wing discs. The larvae were incubated at $29^{\circ} \mathrm{C}$. (h) ap GAL4,UASAppl/UAS-Appl. (i) ap GAL4,UAS-Appl/UAS-Appl; dAPP-BP1 ${ }^{E \times 62}$ + . Apoptosis is greatly enhanced compared to (h). (j) Quantification of TUNEL signals in wing discs. Error bars represent the $95 \%$ confidence interval (2XAPPL = ap GAL4, UAS-Appl/UAS-Appl, 2XAPPL; Ex62 = ap GAL4,UAS-Appl/UAS-Appl; dAPP-BP1 Ex62/ +). (k) APPL expression pattern in wing disc of ap GAL4, UAS-Appl/UAS-Appl 

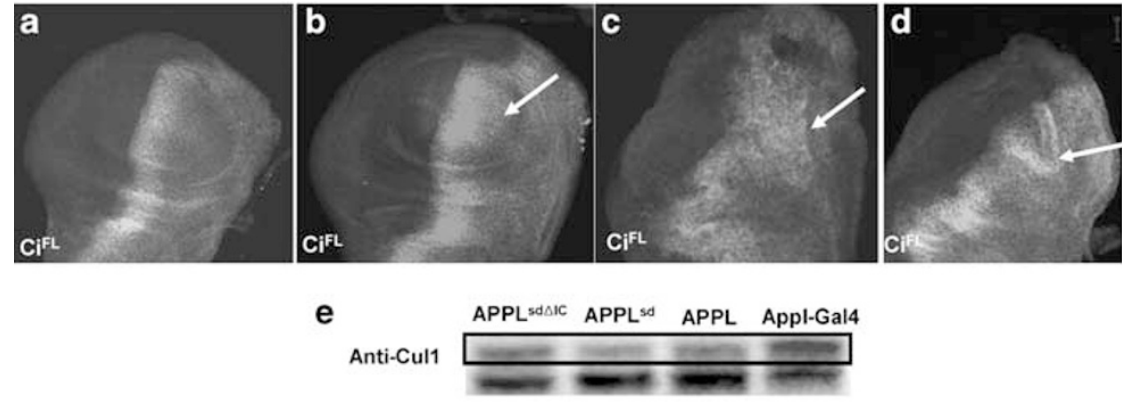

Anti- $\beta$ Tubulin
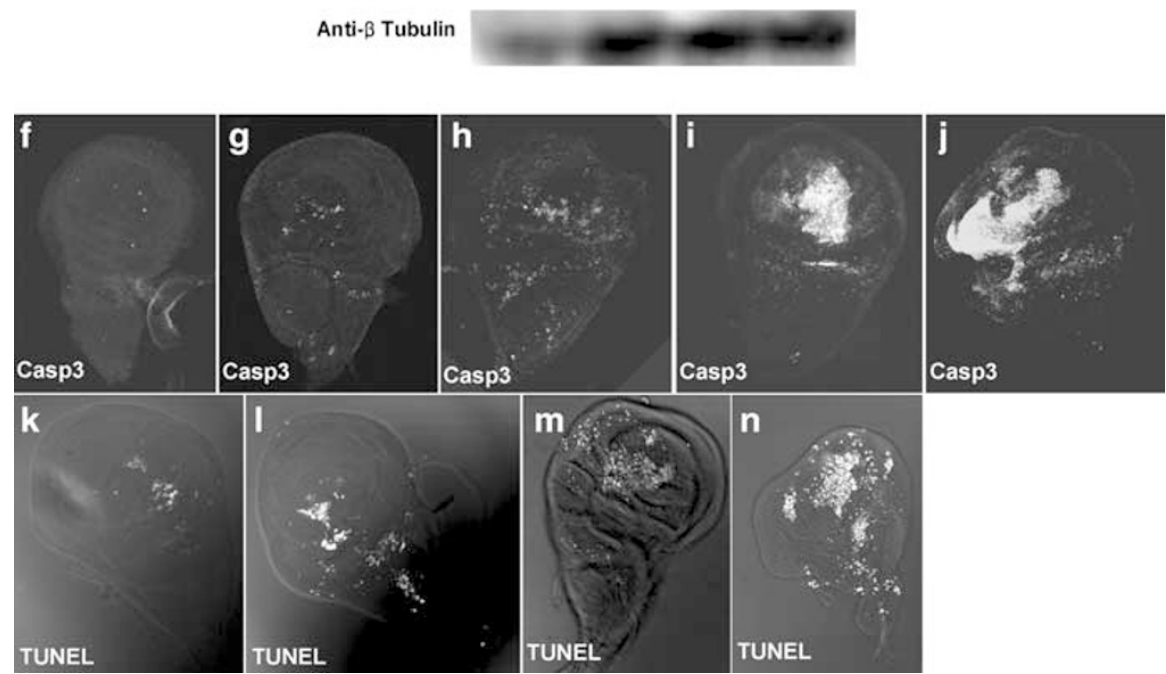

Figure 8 Overexpression of APPL and its derivatives inhibits SCF ligase functions by the neddylation pathway. (a-d) Anti-Ci ${ }^{\mathrm{FL}}$ staining in the third instar larval wing disc imaged with identical confocal microscope settings. All larvae were raised at $29^{\circ} \mathrm{C}$. (a) Full-length Ci level in MS1096. (b) Increased level of full-length Ci in MS1096; UAS-Appl/ UAS-Appl (arrow). (c) MS1096; UAS-App $\beta^{\beta d}+$. C $\mathrm{F}^{\mathrm{FL}}$ staining pattern is broadened and wing disc structure is abnormal. (d) MS1096; UAS-App ${ }^{\beta d \Delta I C /}+$ shows phenotype similar to (c). (e) The neddylation of Cul1 is repressed by overexpression of APPL or APPL derivatives. APPL, APPL ${ }^{\text {sd }}$, and APPL ${ }^{\text {sd } \triangle I C}$ were overexpressed by $A p p l-G A L 4$ at $29^{\circ} \mathrm{C}$. Blacklined box indicates neddylated form of Cul1 protein. ( $(\mathbf{-}-\mathbf{n})$ Third instar larval wing disc incubated at $25^{\circ} \mathrm{C}$. The cell death induced by $A P P L^{\text {sd }}$ and $A P P L^{\text {sd } \triangle I C}$ is enhanced by $d A P P$ BP1 mutation. The amount of apoptosis is more severe when APPL ${ }^{\text {sd }}$ is expressed. (f-j) Immunostaining by anti-active caspase 3 (white). (f) ap-GAL4; $d A P P-B P 11^{E x 62} /+$. (g) ap

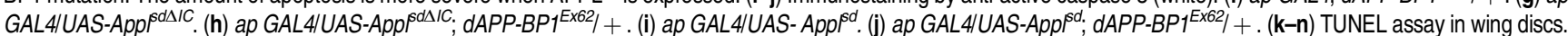

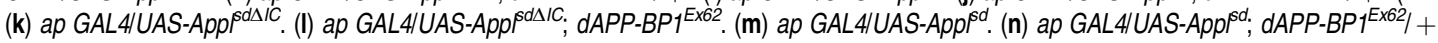

$B P 1$ cannot suppress $A P P L^{s d \Delta / C}$-induced apoptosis, reduction of $d A P P-B P 1$ enhances $A P P L^{s d \Delta / C}$-induced apoptosis (Figure $8 \mathrm{~g}, \mathrm{~h}, \mathrm{k}$ and $\mathrm{l}$ ). This suggests that $A P P L^{s d \Delta / C}$-induced apoptosis is related to neddylation. For $A P P L^{s d \Delta / C}$ and $A P P L^{\text {sd }}$ levels of apoptosis are greater than for $A P P L$. For $A P P L$, increased cell death is observed only when two copies of $A P P L$ are overexpressed and flies are reared at $29^{\circ} \mathrm{C}$ (data not shown, Figure $6 \mathrm{e}-\mathrm{j}$ ). Because expression levels of these UAS lines are not significantly different, ${ }^{23}$ these results demonstrate that cleavage of APPL is important for its regulation. Overexpression of $A P P L$ consistently failed to produce abnormal phenotypes (Figure $7 \mathrm{c}$ ).

\section{Discussion}

In this work, $d A P P-B P 1$ mutants were identified, isolated, and characterized. Immunostaining and immunoblotting of the developing discs showed that loss of dAPP-BP1 blocks the neddylation pathway, suggesting that the role of dAPP-BP1 is similar to that of its mammalian counterpart. A loss-of-function mutant of $d A P P-B P 1$ showed that mutant cells lacking dAPP-
BP1 activity display elevated levels of apoptosis, but no changes in cell proliferation. Imaginal discs are of small size in the $d A P P-B P 1$ null mutant, suggesting that apoptosis plays a significant role in reducing cell number. In $\triangle A P P-B P 1$ null clones, high levels of apoptosis are correlated with loss of photoreceptor neurons in the imaginal eye discs of mutants. Loss of dAPP-BP1 also hinders cell survival in other cell types. Our loss-of-function genetic analysis is in agreement with previous studies showing that mice deficient in the Uba3 gene that encodes a catalytic subunit of NEDD8-activating enzyme died in utero at the peri-implantation stage, with mutant embryos showing apoptosis of their inner cell mass. ${ }^{30}$

Like mammalian APP-BP1, dAPP-BP1 physically interacts with APPL ICD and $d A P P-B P 1$ mutants genetically interact with $A p p{ }^{d}$. The presence of dAPP-BP1 is necessary for attenuated Appl-induced toxicity via the ICD of APPL. The human APP-BP1-binding site is covered with the major APP carboxy-terminal adaptor protein-binding region. ${ }^{27}$ Several mammalian adaptor proteins are conserved in Drosophila. Binding by dAPP-BP1 may regulate an interaction between APPL ICD and other adaptor proteins. Physical interactions 


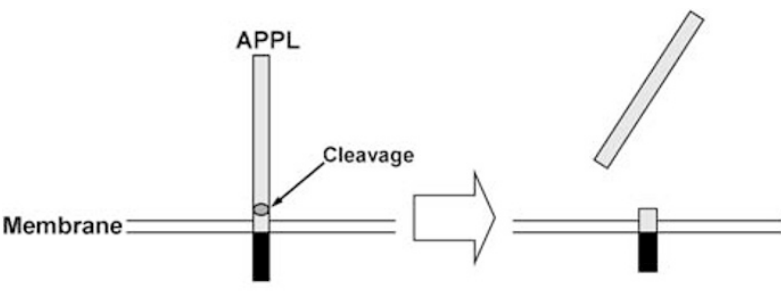

No Apoptosis

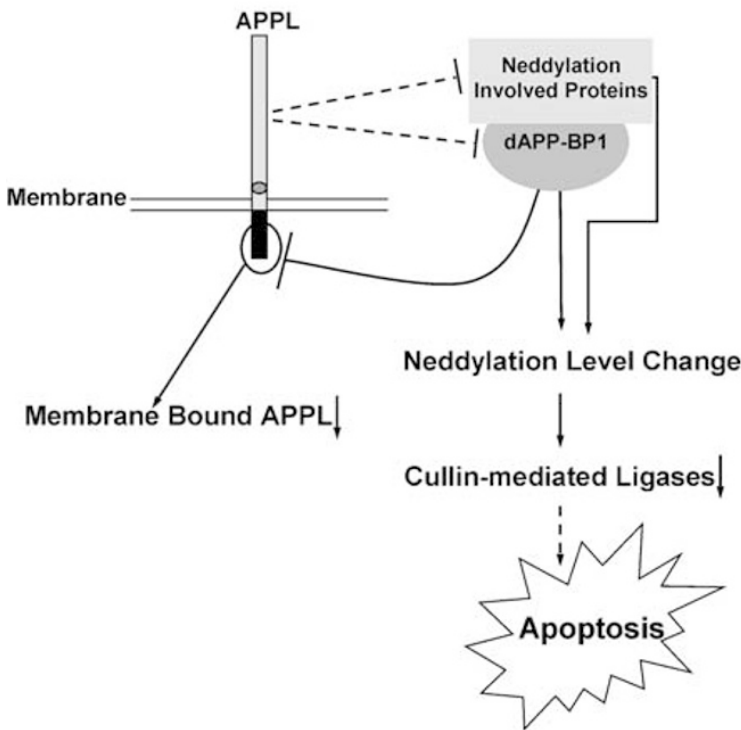

Figure 9 Proposed model for APPL-induced apoptosis (see Discussion for details)

between dAPP-BP1 and APPL may be essential for dAPPBP1-mediated apoptosis suppression, and this suppression results from APPL ICD processing or from signal transduction regulation. Chen et al. ${ }^{4}$ demonstrated that neurons expressing APP or APP (V642I) show an increase in APP-BP1 levels in lipid rafts, which is also observed in the hippocampal region of $A D$ patients. This could explain why an elevated level of membrane APP increases the physical interaction between APP and APP-BP1 required for reducing APP-induced toxicity.

The major target of neddylation is the Cullin family of proteins. ${ }^{7}$ Our study indicates that the membrane-bound form of APPL inhibits SCF ligase functions. Previous studies indicated that the loss of Cul1-mediated SCF ${ }^{\text {slimb }}$ ligase function causes apoptosis and DNA synthesis by abnormal accumulation of E2F. ${ }^{29}$ APP overexpression also causes DNA synthesis and apoptosis in neurons. ${ }^{4}$ Since dAPP-BP1 directly binds to APPL ICD and the mutation suppresses $A p p I^{d}$ phenotypes, we suggest that Appl-induced SCF ligase inhibition is due to changes in the neddylation level of Cullin. Supporting this, Western blot analysis reveals that neddylated Cul1 level is reduced by overexpression of APPL. Also, dAPP. BP1 protein accumulates in $A p p /^{d}$ flies and overexpression of dAPP-BP1 causes a macrochaete-missing phenotype. If APPL is missing, dAPP-BP1 is hyperactivated and the neddylation level of Cullin is increased. When APPL is overexpressed, neddylation is decreased. Increased levels of neddylated Cullin do not necessarily mean enhanced SCF activity. Since CSN (COP9 signalosome) has deneddylase activity, the neddylated Cullin level is greatly increased in CSN mutant cells. ${ }^{31}$ SCF ligase substrates accumulate under these conditions. ${ }^{32}$ Interaction between Cul1 and p120 CAND1 is required for SCF function, ${ }^{33}$ but $\mathrm{p} 120^{\text {CAND1 }}$ binds only to unneddylated Cul1. ${ }^{11,34}$ For this reason, proper levels of neddylation may be important for SCF function.

Some APPL overexpression phenotypes are similar to Cullin mutant phenotypes. Torroja et al. ${ }^{23}$ reported that overexpression of wild-type and mutant forms of APPL in Drosophila motor neurons caused a dramatic increase in synaptic bouton number. Interestingly, recent studies show that synaptic bouton number in motor neuron endings was greatly increased in a Drosophila Cul-5 mutant. ${ }^{35} \mathrm{APPL}^{\text {sd }}$ and APPL ${ }^{\text {sd } \Delta \mathrm{IC}}$ overexpression also caused abnormalities in the adult mushroom bodies, with the lobes being less uniform and having fuzzy boundaries. ${ }^{26}$ APPL overexpression has been shown to induce post-developmental neurite arborization. ${ }^{36}$ Similarly, Zhu et al. ${ }^{37}$ showed the cell-autonomous involvement of Drosophila Cul3 in axonal arborization and dendritic elaboration of mushroom body neurons. The neddylation of cullins is important for their proper functioning, so that the APPL overexpression phenotypes of these reports may be directly related to reduced neddylation.

The extracellular region of APPL can partially inhibit the neddylation pathway when the transmembrane domain is intact. This repression is enhanced by the ICD. The extracellular domain of APP cannot interact physically with APP-BP1; consequently, it is possible that dAPP-BP1 is not the target of APPL-induced inhibition. However, the close correlation between their respective levels suggests that APPL could be targeting dAPP-BP1. We propose two modes of APPL regulation in Drosophila development (Figure 9). If APPL is cleaved at the extracellular cleavage site, it does not cause apoptosis. When the membrane-bound form of APPL increases, it interacts antagonistically with dAPP-BP1 and/or other neddylation proteins. As a result of this repression, the level of neddylated cullins, SCF ligase activity, and other cullin-mediated ligase activities are all reduced. The reduction of ligase activity then causes apoptosis. Direct interaction between dAPP-BP1 and APPL ICD can attenuate membranebound APPL functions. Mechanisms underlying the APP pathway are closely conserved in Drosophila and in mammals. Consequently, the study of Drosophila Appland $d A P P$ $B P 1$ can contribute substantially to the elucidation of APP function.

\section{Materials and Methods}

Immunocytochemistry of larval discs. Staged larvae are dissected in $1 \times$ PBS. Dissected head cuticles are incubated in fixation solution $(0.1 \mathrm{M}$ PIPES, pH 6.9, 1 mM EDTA, 1.0\% Triton X-100, $2 \mathrm{~mm} \mathrm{MgSO}_{4}, 1 \%$ EM grade formaldehyde) for $30 \mathrm{~min}$. After incubating in blocking solution $(50 \mathrm{~mm}$ Tris-Cl, pH 6.8, $150 \mathrm{mM}$ $\mathrm{NaCl}, 0.5 \% \mathrm{NP}-40,5 \mathrm{mg} / \mathrm{ml} \mathrm{BSA}, 0.01 \%$ Thimerosal) at $4^{\circ} \mathrm{C}$ for $1 \mathrm{~h}$, head cuticles are transferred to each primary antibody in wash solution $(50 \mathrm{~mm} \mathrm{Tris-Cl,} \mathrm{pH} 6.8$, $150 \mathrm{~mm} \mathrm{NaCl}, 0.5 \% \mathrm{NP}-40,1 \mathrm{mg} / \mathrm{ml} \mathrm{BSA}, 0.01 \%$ Thimerosal) and incubated at $4^{\circ} \mathrm{C}$ overnight. After washing four times in wash solution, head cuticles are incubated with secondary antibody at room temperature for $2 \mathrm{~h}$. After washing three times with wash solution, head cuticles are dissected in antifade solution. Primary antibodies 
were mouse anti-Armadillo (N27A1, 1:50, lowa Hybridoma Bank), rat anti-elav (7E8A10, 1:500, lowa Hybridoma Bank), rabbit anti- $\beta$-gal (1:1000, Sigma), mouse anti-GFP (1:200, Sigma), rat anti-Ci (2A1, 1:3), ${ }^{38}$ rabbit anti-Cul1 (1:250, Zymed), rabbit anti-cleaved (activated) caspase 3 (1:50, Cell Signaling Technology), rabbit anti-phospho-histone H3 (1:500, Upstate Biotechnology), rabbit anti-APPL (Ab952, $1: 500),{ }^{21}$ and mouse anti-Myc (1:200, Roche). Secondary antibodies coupled to the appropriate fluorophore (FITC, Сy3, or Cy5; Jackson Immunoresearch Laboratories) were diluted to 1:200 in wash solution. All confocal images were taken on an LSM510 (Carl Zeiss). Images were processed in Adobe Photoshop.

DNA constructs for germline transformation. Wild-type dAPP-BP1 full-length CDNAs were cloned into the pUAST vector and PUAST-HA vector as EcoRI-Xbal fragments. P-element-mediated germline transformation was performed according to described methods. ${ }^{39}$ The parental strain for all germline transformations was $w^{1118}$. Flies bearing autosomal transgenes were identified and used for all analyses.

Fly strains. All stocks were maintained and raised under standard conditions. Oregon $\mathrm{R}$ wild-type and $w^{1118}$ were used as control strains. App ${ }^{d}$, Appl-GAL4, UAS$A p I^{+}, A p p{ }^{\beta d}$, and $\left.A p p\right|^{s}$ were gifts from Kalpana White ${ }^{22}$ and ap-GAL4 was a gift from P Adler. UAS-App $\left.\right|^{\delta \Delta / C}$ was provided by Renato Paro. The MS1096 stock is as described in Capdevila and Guerrero. ${ }^{40}$ The Cul1 allele Cul1 ${ }^{E x}$ was described previously. ${ }^{13}$ The Bloomington Stock Center (Bloomington, IN; http:// flystocks.bioindiana.edu/) provided other fly stocks.

TUNEL assay. TUNEL assays were performed using the Roche in situ cell death detection kit (fluorescein, Catalog number 1684 795). In brief, imaginal discs were fixed with fixation solution (0.1 M PIPES, pH 6.9, 1 mM EDTA, 1.0\% Triton X$100,2 \mathrm{mM} \mathrm{MgSO}_{4}, 1 \% \mathrm{EM}$ grade formaldehyde) for $30 \mathrm{~min}$ and washed with PBT (PBS, $0.1 \%$ Triton $\mathrm{X}-100$ ) four times for $10 \mathrm{~min}$. Discs were then washed twice for 5 min with PBT $5 \times$ (PBS, $0.5 \%$ Triton X-100) and transferred to a reaction buffer for $10 \mathrm{~min}$, followed by working-strength reaction buffer containing terminal deoxynucleotide transferase. The discs were incubated with the enzyme in a humidified chamber at $37^{\circ} \mathrm{C}$ for $2 \mathrm{~h}$. The reaction was stopped by transferring the discs into PBT $5 \times$ and discs were then rinsed in three changes of PBT. Discs were then immunoreacted with antibodies or directly imaged by confocal microscopy.

Clonal analysis. Mitotic recombination clones were induced $48+3 \mathrm{~h}$ after egg laying (AEL) in staged larvae by heat shock at $37^{\circ} \mathrm{C}$ for $80 \mathrm{~min}$. Larval genotypes were: P\{hsFLP\}22 $y^{1} w^{*} ;$ P $\{$ arm-lacZ.V $\}$ 70C P\{neoFRT\} 80B/dAPPBP ${ }^{\text {ex62 }} P\{$ neo

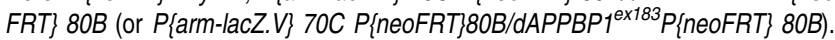
Discs were dissected at $110+3 \mathrm{~h}$ AEL, fixed in fixation solution, and stained with anti- $\beta$-galactosidase antibody to mark the clones. The following flies were used to generate $d A P P-B P 1$ clones in the eye disc: $y^{d 2} w^{1118} P\{$ ey-FLP.N\}2; P $\{U b i-G F P\}$ 61EF P\{neoFRT\}80B/dAPPBP1 ${ }^{E \times 62} \quad P\{$ neoFRT $\} 80 B$ (or P\{Ubi-GFP $61 E F$ $P\{$ neofRT $\} 80 B / d A P P B P 1^{E x 183} P\{$ neoFRT $\left.\} 80 B\right)$ and $y^{d 2} w^{1118} P\{$ ey-FLP.N\}2; $P\{\pi M\} 75 C P$ \{neoFRT\} 80B/dAPP-BP1 ${ }^{E \times 62}$ P\{neoFRT\}80B (or $P\{\pi M\} 75 C$ $P\{$ neoFRT $\} 80 B / d A P P B P 1{ }^{E x 183} P\{$ neoFRT $\left.\} 80 B\right)$. Clone areas were measured using Adobe Photoshop 6.0.

Western blotting and antibody generation. A $\triangle A P P-B P 1$ CDNA (corresponding to amino-acid residues 147-299) was cloned into $\mathrm{pET}-15 \mathrm{~b}$ vector that encodes a fusion protein with $6 \times$ His tags. The fusion protein was expressed in Escherichia coli and purified with His.Bind Resin (Novagen). Polyclonal antibodies were produced by immunizing guinea pigs using the purified fusion protein as antigen (KOMA Biotech, Korea).

Larval brain and eye discs or adult heads were collected in $2 \times$ SDS sample buffer and boiled for $10 \mathrm{~min}$. The extracts were then run on a 3-8\% Tris-acetate gel (Invitrogen) or 12\% Tris-glycine gel. Western blotting was performed according to standard protocol. Primary antibodies used were rabbit anti-Cul1 (1:750; Zymed), rabbit anti-APPL (1:4000; gift from K White), guinea-pig anti-dAPP-BP1 (1:500), and $\beta$-tubulin ( $E 7$, lowa Hybridoma Bank, 1:2000). After incubation with HRP-coupled secondary antibodies (goat anti-rat diluted 1:3000 and goat anti-rabbit diluted 1:3000; Pierce), blots were visualized using a chemiluminescent detection kit (ECL; Amersham Biosciences).

RT-PCR and quantitative RT-PCR. For RT-PCR, total RNA was prepared using TRIzol (Invitrogen) and reverse transcribed using M-MLV reverse transcriptase (Promega). PCR was performed using dAPP-BP1 specific primer including an intron with forward sequence $5^{\prime}$-CATATGCGGTTATGGGAGCTTAAC$3^{\prime}$ and reverse sequence $5^{\prime}$-GGATCCCTGCTTAAGGTACTCCTG- $3^{\prime}$ to obtain a product of $640 \mathrm{bp}$. The GAP-DH control was amplified with the forward primer 5'-GTCAA CGATCCCTTCATCGA-3' and reverse primer 5'-TGTACGATAGTTGTGGCTAG- ${ }^{\prime}$. For quantitative RT-PCR, total RNA was isolated from each stage of Oregon R. First-strand cDNA was synthesized from $3 \mu \mathrm{g}$ of total RNA with a total cycle number of 25 . For control, rp49 primers were used.

GST pull-down analysis. ${ }^{35} \mathrm{~S}$-labeled dAPP-BP1 was synthesized in vitro using pSP64-AT09990 (dAPP-BP1 expressed sequence tag (EST)), TNT QuickCoupled Transcription/Translation system (Promega), and L- $\left[{ }^{35} \mathrm{~S}\right]$ methionine (Amersham Biosciences) according to the manufacturer's protocol. BL21 cells containing GST and GST-APPL C63 (GST-tagged APPL, carboxy-terminal 63 amino-acid residues) expression plasmids (pGEX 6P-1) were grown in LB media. Expression of fusion proteins was induced by treating cells with $0.5 \mathrm{~mm}$ isopropyl-1. thio-beta-D-galactopyranoside for $12 \mathrm{~h}$ at $20^{\circ} \mathrm{C}$. GST fusion protein was purified by the batch GST Purification Module (Amersham Biosciences). GST-APPL C63 fusion protein and GST were isolated by using glutathione Sepharose 4 fast flow beads. The immobilized GST and GST-APPL C63 protein beads were washed with phosphate-buffered saline and incubated with ${ }^{35} \mathrm{~S}$-labeled dAPP-BP 1 at $4{ }^{\circ} \mathrm{C}$ for $12 \mathrm{~h}$ in binding buffer ( $20 \mathrm{~mm}$ Hepes, $0.1 \mathrm{~mm}$ EDTA, $10 \mathrm{~mm} \mathrm{KCL}, 10 \%$ glycerol, $0.4 \%$ Triton-X 100 and Sigma protease inhibitor cocktail). Beads were washed three times with buffer and subjected to SDS-polyacrylamide gel electrophoresis and autoradiography.

\section{Acknowledgements}

We thank Daria Hekmat-Scafe, Jeff Tan, Ed Glasscock, Shefa Gordon, and Juan Song for discussion and contributions to this work. The research was supported by a grant (M103KV010002 04K2201 00230) from Brain Research Center of the 21st Century Frontier Research Program funded by the Ministry of Science and Technology, the Republic of Korea to JY, and grants from the NIH and the Epilepsy Foundation to MT

1. Chow N, Korenberg JR, Chen X-N, Neve RL. APP-BP1, a novel protein that binds to the carboxyl-terminal region of the amyloid precursor protein. J Biol Chem 1996; 271: 1133911346

2. Hochstrasser M. Evolution and function of ubiquitin-like protein-conjugation systems. Nat Cell Biol 2000; 2: E153-E157.

3. Chen Y, McPhie DL, Hirschberg J, Neve RL. The amyloid precursor protein-binding protein APP-BP1 drives the cell cycle through the S-M checkpoint and causes apoptosis in neurons. J Biol Chem 2000; 275: 8829-8835.

4. Chen Y, Liu W, McPhie DL, Hassinger L, Neve RL. APP-BP1 mediates APP-induced apoptosis and DNA synthesis and is increased in Alzheimer's disease brain. $J$ Cell Biol 2003; 163: 27-33

5. Pan ZQ, Kentsis A, Dias DC, Yamoah K, Wu K. Nedd8 on cullin: building an expressway to protein destruction. Oncogene 2004; 23: 1985-1997.

6. Xirodimas DP, Saville MK, Bourdon JC, Hay RT, Lane DP. Mdm2-mediated NEDD8 conjugation of p53 inhibits its transcriptional activity. Cell 2004; 118: 83-97.

7. Deshaies RJ. SCF and Cullin/Ring H2-based ubiquitin ligases. Annu Rev Cell Dev Biol 1999; 15: 435-467.

8. Karin M, Ben-Neriah Y. Phosphorylation meets ubiquitination: the control of NF-[kappa]B activity. Annu Rev Immunol 2000; 18: 621-663.

9. Kondo K, Kaelin JWG. The von Hippel-Lindau tumor suppressor gene. Exp Cell Res 2001; 264: 117-125.

10. Ohh M, Kim WY, Moslehi JJ, Chen Y, Chau V, Read MA et al. An intact NEDD8 pathway is required for Cullin-dependent ubiquitylation in mammalian cells. EMBO Rep 2002; 3: $177-$ 182

11. Liu J, Furukawa M, Matsumoto T, Xiong Y. NEDD8 modification of CUL1 dissociates p120CAND1, an inhibitor of CUL1-SKP1 binding and SCF ligases. Mol Cell 2002; 10 1511-1518.

12. Wu K, Chen A, Tan P, Pan ZQ. The Nedd8-conjugated ROC1-CUL1 core ubiquitin ligase utilizes Nedd8 charged surface residues for efficient polyubiquitin chain assembly catalyzed by Cdc34. J Biol Chem 2002; 277: 516-527.

13. Ou CY, Lin YF, Chen YJ, Chien CT. Distinct protein degradation mechanisms mediated by Cul1 and Cul3 controlling Ci stability in Drosophila eye development. Genes Dev 2002; 16 2403-2414.

14. Mori F, Nishie M, Piao YS, Kito K, Kamitani T, Takahashi H et al. Accumulation of NEDD8 in neuronal and glial inclusions of neurodegenerative disorders. Neuropathol App Neurobiol 2005; 31: 53-61. 
15. Rosen DR, Martin-Morris L, Luo LQ, White K. A Drosophila gene encoding a protein resembling the human beta-amyloid protein precursor. Proc Natl Acad Sci USA 1989; 86 2478-2482.

16. Kang J, Lemaire HG, Unterbeck A, Salbaum JM, Masters CL, Grzeschik KH et al. The precursor of Alzheimer's disease amyloid A4 protein resembles a cell-surface receptor. Nature 1987; 325: 733-736.

17. Luo LQ, Martin-Morris LE, White K. Identification, secretion, and neural expression of APPL, a Drosophila protein similar to human amyloid protein precursor. J Neurosci 1990; 10: $3849-3861$.

18. Sisodia SS. Cellular and molecular genetic approaches in Alzheimer's disease. Neurobiol Aging 1994; 15: S135-S137.

19. Slunt HH, Thinakaran G, Von Koch C, Lo AC, Tanzi RE, Sisodia SS. Expression of ubiquitous, cross-reactive homologue of the mouse beta-amyloid precursor protein (APP). J Biol Chem 1994; 269: 2637-2644.

20. Luo L, Tully T, White K. Human amyloid precursor protein ameliorates behavioral deficit of flies deleted for Appl gene. Neuron 1992; 9: 595-605.

21. Torroja L, Luo L, White K. APPL, the Drosophila member of the APP-family, exhibits differential trafficking and processing in CNS neurons. J Neurosci 1996; 16: 4638-4650.

22. Torroja L, Chu H, Kotovsky I, White K. Neuronal over-expression of APPL, the Drosophila homologue of the amyloid precursor protein (APP), disrupts axonal transport. Curr Biol 1999a; 9: 489-493.

23. Torroja L, Packard M, Gorczyca M, White K, Budnik V. The Drosophila beta-amyloid precursor protein homolog promotes synapse differentiation at the neuromuscular junction. J Neurosci 1999b; 15: 7793-7803.

24. Gunawardena S, Goldstein LSB. Disruption of axonal transport and neuronal viability by amyloid precursor protein mutations in Drosophila. Neuron 2001; 32: 389-401.

25. Tschape JA, Hammerschmied C, Muhlig-Versen M, Athenstaedt K, Daum G, Kretzschma $D$. The neurodegeneration mutant lochrig interferes with cholesterol homeostasis and Appl processing. EMBO J 2002; 21: 6367-6376.

26. Li Y, Liu T, Peng Y, Yuan C, Guo A. Specific functions of Drosophila amyloid precursor-like protein in the development of nervous system and nonneural tissues. J Neurobiol 2004; 61 343-358.

27. King GD, Scott Turner R. Adaptor protein interactions: modulators of amyloid precursor protein metabolism and Alzheimer's disease risk? Exp Neurol 2004; 185: 208-219.
28. Merdes G, Soba P, Loewer A, Bilic MV, Beyreuther K, Paro R. Interference of human and Drosophila APP and APP-like proteins with PNS development in Drosophila. EMBO J 2004; 23: 4082-4095.

29. Hériché JK, Ang D, Bier E, O'Farrell PH. Involvement of an SCFSImb complex in timely elimination of E2F upon initiation of DNA replication in Drosophila. BMC Genet 2003; 4: 9 21

30. Tateishi K, Omata M, Tanaka K, Chiba T. The NEDD8 system is essential for cell cycle progression and morphogenetic pathway in mice. J Cell Biol 2001; 155: 571-579.

31. Bech-Otschir D, Seeger M, Dubiel W. The COP9 signalosome: at the interface between signal transduction and ubiquitin-dependent proteolysis. J Cell Sci 2002; 115: 467-473.

32. Cope GA, Deshaies RJ. COP9 signalosome: a multifunctional regulator of SCF and other cullin-based ubiquitin ligases. Cell 2003; 114: 663-671.

33. Feng S, Shen Y, Sullivan JA, Rubio V, Xiong Y, Sun TP et al. Arabidopsis CAND1, an unmodified CUL1-interacting protein, is involved in multiple developmental pathways controlled by ubiquitin/proteasome-mediated protein degradation. Plant Cell 2004; 16 : 1870-1882.

34. Zheng J, Yang X, Harrell JM, Ryzhikov S, Shim EH, Lykke-Andersen K et al. CAND1 binds to unneddylated CUL1 and regulates the formation of SCF ubiquitin E3 ligase complex. Mol Cell 2002; 10: 1519-1526.

35. Ayyub C, Sen A, Gonsalves F, Badrinath K, Bhandari P, Shashidhara LS et al. Cullin-5 plays multiple roles in cell fate specification and synapse formation during Drosophila development. Dev Dyn 2005; 232: 865-875.

36. Leyssen M, Ayaz D, Hebert SS, Reeve S, De Strooper B, Hassan BA. Amyloid precursor protein promotes post-developmental neurite arborization in the Drosophila brain. EMBO J 2005; 24: 2944-2955.

37. Zhu S, Perez R, Pan M, Lee T. Requirement of Cul3 for axonal arborization and dendritic elaboration in Drosophila mushroom body neurons. J Neurosci 2005; 25: 4189-4197.

38. Motzny CK, Holmgren R. The Drosophila cubitus interruptus protein and its role in the wingless and hedgehog signal transduction pathways. Mech Dev 1995; 1: 137-150.

39. Spradling A, Rubin GM. Genetic transformation of Drosophila with transposable element vectors. Science 1982; 4570: 348-353.

40. Capdevila J, Guerrero I. Targeted expression of the signaling molecule decapentaplegic induces pattern duplications and growth alterations in Drosophila wings. EMBO J 1994; 13: 4459-4468.

Supplementary Information accompanies the paper on Cell Death and Differentiation website (http://www.nature.com/cdd) 